\title{
Search for CAC-integrable homogeneous quadratic triplets of quad equations and their classification by BT and Lax
}

\author{
Jarmo Hietarinta* \\ Department of Physics and Astronomy \\ University of Turku, FIN-20014 Turku, Finland
}

March 12, 2019

\begin{abstract}
We consider two-dimensional lattice equations defined on an elementary square of the Cartesian lattice and depending on the variables at the corners of the quadrilateral. For such equations the property often associated with integrability is that of "multidimensional consistency" (MDC): it should be possible to extend the equation from two to higher dimensions so that the embedded two-dimensional lattice equations are compatible. Usually compatibility is checked using "ConsistencyAround-a-Cube" (CAC). In this context it is often assumed that the equations on the six sides of the cube are the same (up to lattice parameters), but this assumption was relaxed in the classification of Boll [3]. We present here the results of a search and classification of homogeneous quadratic triplets of multidimensionally consistent lattice equations, allowing different equations on the three orthogonal planes (hence triplets) but using the same equation on parallel planes. No assumptions are made about symmetry or tetrahedron property. The results are then grouped by subset/limit properties, and analyzed by the effectiveness of their Bäcklund transformations, or equivalently, by the quality of their Lax pair (fake or not).
\end{abstract}

\section{Introduction}

\subsection{General setting}

When we discuss integrable equations, continuous or discrete, there is always the question of which definition of integrability should be used. Unfortunately at this time it is still not possible to give a universal definition of integrability. This is because although

*E-mail: jarmo.hietarinta@utu.fi 
integrability can be discussed for various different classes of equations, it manifests itself in different form depending on the class. In place of an elusive universal definition, we do have many different specific properties that are associated with equations that are considered integrable. These properties depend on the class of equations. ${ }^{1}$ However, there are some properties that can be applied to a variety of classes of equations, such as behavior around singularities, existence of a Lax pair with spectral parameter, or the existence of sufficient number of conservation laws, and these have sometimes been elevated as definitions of integrability, while some other proposed definitions can only be taken as indicators, or necessary conditions.

In this paper we consider difference equations defined on an elementary square of the Cartesian lattice, with the dynamical variables located on the corners of the square, so called quadrilateral equations. The following basic assumptions are made:

\section{Definition 1 (Acceptable equations) :}

1. The equation depends on all corner variables of the quadrilateral.

2. The equation is affine linear in each corner variable.

3. The equation is irreducible.

In this paper we assume furthermore that the equations are homogeneous quadratic.

Within the present context of quadrilateral equations there are again several properties that are strongly associated with integrability. One is obtained using algebraic entropy [11], that is, the growth of complexity under evolution: If growth is linear the equation is linearizable, if the growth is polynomial the equation is integrable, if the growth is exponential the equation shows chaotic behavior. Other information is obtained from symmetry analysis [9].

The properties just mentioned involve analysis on the $2 \mathrm{D}$ lattice, but there is also a criterion that is based on a multidimensional lattice. It is well known that soliton equations come in hierarchies, with infinite number of different evolutionary times, and in the context of lattice equations this has been associated with multidimensionality [10].

In such an approach the original $2 \mathrm{D}$ quadrilateral equation is extended into $\mathbb{Z}^{3}$ by introducing accompanying compatible equations on the other 2D-planes of the 3D-space. In the strictest version the accompanying equations are obtained from the original just by changing some lattice variables and parameters. This approach was used by Adler, Bobenko and Suris (together with the additional assumptions of symmetry and the "tetrahedron property" (TET)) in order to obtain a classification, the "ABS list" [2], which has been important in resurrecting the study of integrable lattice equations. A more relaxed version is to allow completely different quad-equations on the three different $\mathbb{Z}^{2}$ lattice planes, this approach was taken by Boll in his classification [3], where the tetrahedron condition was also used extensively. Even more generally, the accompanying equations could also live in bigger stencils (this is needed if the Lax matrix is bigger that $2 \times 2$.)

\footnotetext{
${ }^{1}$ For example the existence of $N$-soliton solutions for $(1+1)$ dimensional PDE's is strongly associated with integrability, but it does not even make sense for Hamiltonian mechanical systems.
} 
In any case the 3D system of equations must be compatible. If all equations are quad equations, the compatibility condition is called "Consistency-Around-a-Cube" (CAC) and is elaborated below. One statement relating 2D-integrability and CAC is that if an equation has been found to be integrable by a $2 \mathrm{D}$ condition, then such a compatible extension should be possible. ${ }^{2}$

In the present work we take completely free acceptable (by Definition 1) quadratic equations on the three $\mathbb{Z}^{2}$ lattices of $\mathbb{Z}^{3}$ and classify those that have CAC.

\subsection{Detailed formulation}

For consistency analysis we consider a 3D cube and assign equations on each side of the cube. Furthermore we will extend the consideration from one cube to the full $\mathbb{Z}^{3}$ lattice and embed the equation as follows:

1. On a given $\mathbb{Z}^{2}$ plane all elementary squares have equations of the same form (that is, only the corner variables change corresponding to the location). The coefficients of the equation may depend on the two lattice parameters associated with the plane but not on the location.

2. When extended to the $\mathbb{Z}^{3}$ lattice, the quadrilaterals on parallel planes all carry the same equation but intersecting planes may have different equations.

In $\mathbb{Z}^{2}$ the corner variables are usually notated as $x_{n, m}$ where $(n, m)$ gives the locations on the Cartesian plane, while in $\mathbb{Z}^{3}$ the variables are indexed as $x_{n, m, k}$. When dealing with a specific quadrilateral or cube we use shorthand notation

$$
\begin{aligned}
& x_{n, m, k}=x, x_{n+1, m, k}=\widetilde{x}, x_{n, m+1, k}=\widehat{x}, x_{n, m, k+1}=\bar{x} \\
& x_{n+1, m+1, k}=\widehat{\widetilde{x}}, x_{n+1, m, k+1}=\overline{\widetilde{x}}, x_{n, m+1, k+1}=\overline{\widehat{x}}, x_{n+1, m+1, k+1}=\overline{\widehat{\widehat{x}}}
\end{aligned}
$$

An acceptable quadratic quadrilateral equation can then be written as

$$
Q_{12}(x, \widetilde{x}, \widehat{x}, \widehat{\widetilde{x}} ; p, q):=x \widetilde{x} c_{1}+\widetilde{x} \widehat{x} c_{2}+\widehat{x} \widehat{\widetilde{x}} c_{3}+x \widehat{\widetilde{x}} c_{4}+x \widehat{x} c_{5}+\widetilde{x} \widehat{\widetilde{x}} c_{6}=0
$$

where the $c_{j}$ may depend on the lattice variables $p, q$, which are associated with the $n, m$ or tilde and hat directions, respectively. The lattice variable for $k$ or bar direction is $r$. For the consistency cube we need $Q_{12}(x, \widetilde{x}, \widehat{x}, \widehat{\widetilde{x}} ; p, q)=0, Q_{23}(x, \widehat{x}, \bar{x}, \overline{\widehat{x}} ; q, r)=0$, and $Q_{31}(x, \bar{x}, \widetilde{x}, \overline{\widetilde{x}} ; r, p)=0$, that is

$$
\begin{aligned}
x \widetilde{x} c_{1}(p, q)+\widetilde{x} \widehat{x} c_{2}(p, q)+\widehat{x} \widehat{\widetilde{x}} c_{3}(p, q)+x \widehat{\widetilde{x}} c_{4}(p, q)+x \widehat{x} c_{5}(p, q)+\widetilde{x} \widehat{\widetilde{x}} c_{6}(p, q)=0, \\
x \widehat{x} c_{1}(q, r)+\widetilde{x} \bar{x} c_{2}(q, r)+\bar{x} \widehat{\widehat{x}} c_{3}(q, r)+x \widehat{\widehat{x}} c_{4}(q, r)+x \bar{x} c_{5}(q, r)+\widehat{x} \bar{x} c_{6}(q, r)=0, \\
x \bar{x} c_{1}(r, p)+\bar{x} \widetilde{x} c_{2}(r, p)+\widetilde{x} \overline{\widetilde{x}} c_{3}(r, p)+x \overline{\widetilde{x}} c_{4}(r, p)+x \widetilde{x} c_{5}(r, p)+\bar{x} \bar{x} c_{6}(r, p)=0,
\end{aligned}
$$

\footnotetext{
${ }^{2}$ For example, the $Q_{V}$ equation found by Viallet using algebraic entropy analysis [12] has many free parameters and when that equation is extended to $3 \mathrm{D}$ one is led to the parameterization of Adler's $Q_{4}$ equation [1].
} 

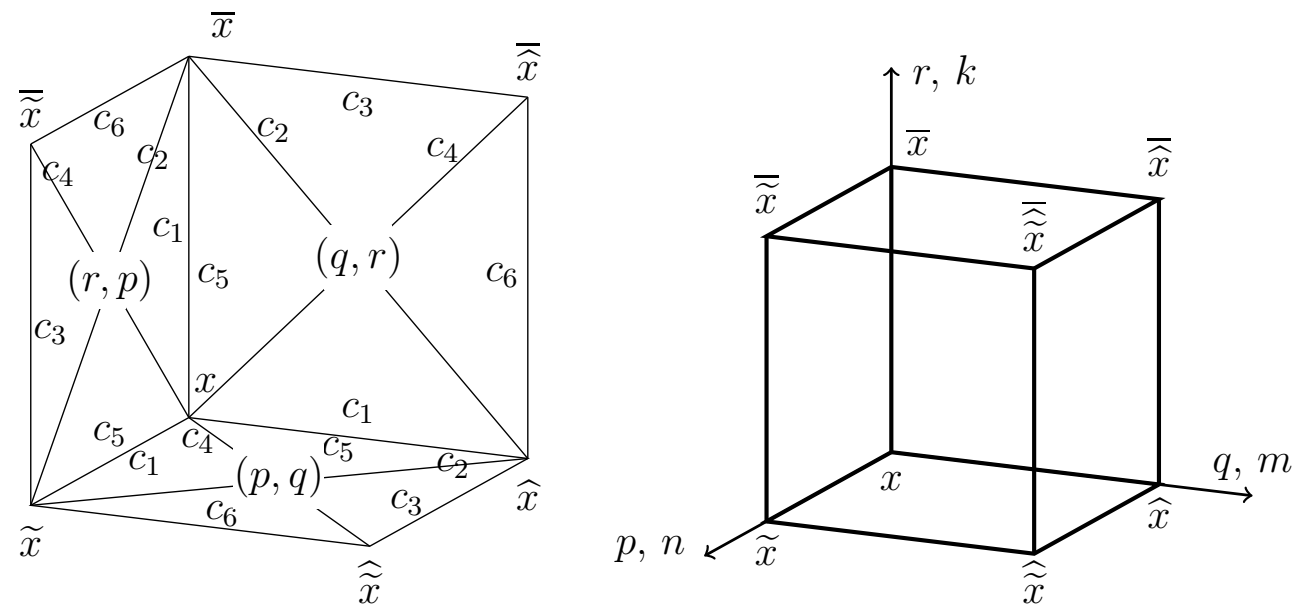

Figure 1: The consistency cube. The picture on the right gives the location of the variables, lattice directions and parameters. The picture on the left explains the coefficients: the variables of a product are connected by a line, adjacent to which is given the name of the corresponding coefficient function. The six functions within a quadrilateral depend on the lattice parameters given at the center of the quadrilateral.

respectively. We also need their shifts. When writing this set of equations we have used cyclic convention $p \rightarrow q \rightarrow r \rightarrow p$ and $\sim \rightarrow^{\wedge} \rightarrow-\rightarrow^{\sim}$, see also Figure 1 . Note that the names of the lattice variables in $c_{i}$ are also used for function names. That means, for example, that there is no $c_{1}(x, y)$ from which $c_{1}(p, q)$ and $c_{1}(q, r)$ could be obtained, rather $c_{1}(p, q)$ and $c_{1}(q, r)$ are independent functions.

Under the assumptions above the consistency conditions arise as follows: The equations on the faces of the consistency cube are given by:

$$
\begin{aligned}
\text { bottom: } & Q_{12}(x, \widetilde{x}, \widehat{x}, \widehat{\widetilde{x}} ; p, q)=0 . & \text { top: } & Q_{12}(\bar{x}, \overline{\widetilde{x}}, \overline{\widehat{x}}, \overline{\widehat{x}} ; p, q)=0, \\
\text { back: } & Q_{23}(x, \widehat{x}, \bar{x}, \overline{\widehat{x}} ; q, r)=0, & \text { front: } & Q_{23}(\widetilde{x}, \widehat{\widetilde{x}}, \overline{\widetilde{x}}, \overline{\widehat{x}} ; q, r)=0, \\
\text { left: } & Q_{31}(x, \bar{x}, \widetilde{x}, \overline{\widetilde{x}} ; r, p)=0, & \text { right: } & Q_{31}(\widehat{x}, \widehat{\widehat{x}}, \widehat{\widehat{x}}, \overline{\widehat{x}} ; r, p)=0
\end{aligned}
$$

We need precisely four initial values to compute the other values, three of the initial values should be on one quadrilateral and the fourth somewhere else. The standard way (there are others) is to take $x, \widetilde{x}, \widehat{x}, \bar{x}$ as the initial values and solve the LHS equations of (3) for $\widehat{\widetilde{x}}, \overline{\widehat{x}}$ and $\overline{\widetilde{x}}$ and substitute them to the RHS. Then from each of the three RHS equations we can solve for $\overline{\widehat{x}}$ and the results should be the same, therefore we get two conditions for consistency.

\subsection{Organization of the search}

The condition that the different ways to compute $\overline{\widehat{x}}$ provide the same result leads to a set of equations which are polynomials in the initial values $x, \widetilde{x}, \widehat{x}, \bar{x}$. The coefficient of 
every monomial $x^{\alpha} \widetilde{x}^{\beta} \widehat{x}^{\gamma} \bar{x}^{\delta}$ has to vanish separately and this yields polynomial equations on $c_{i}(p, q), c_{i}(q, r), c_{i}(r, p), \forall i=1, \ldots, 6$.

In analyzing the ensuing equations the solution process branches depending on whether some coefficient function is zero or not. It is not easy to keep track of such branching and therefore we choose here a different approach.

We will do a pre-analysis by setting some coefficients in (1) to zero. For that purpose let us write (1) as

$$
\iota_{1} x \widetilde{x} c_{1}+\iota_{2} \widetilde{x} \widehat{x} c_{2}+\iota_{3} \widehat{x} \widehat{\widetilde{x}} c_{3}+\iota_{4} x \widehat{\widetilde{x}} c_{4}+\iota_{5} x \widehat{x} c_{5}+\iota_{6} \widetilde{x} \widehat{\widetilde{x}} c_{6}=0
$$

where $\iota_{j} \in\{0,1\}$ while $c_{j}$ are all nonzero. By various choices of $\iota_{j}$ we can have $2^{6}=64$ equations, but by the conditions in Definition 1 this actually yields 37 equations. In order to keep track of the different cases it is convenient to name the equations by the list $\left\{\iota_{1}, \iota_{2}, \iota_{3}, \iota_{4}, \iota_{5}, \iota_{6}\right\}$ and if we consider this as a binary number then we can construct also the corresponding decimal number: $N=\iota_{1}+2 \iota_{2}+4 \iota_{3}+8 \iota_{4}+16 \iota_{5}+32 \iota_{6}$. Then we have for example

$$
x \widetilde{x} c_{1}+\widetilde{x} \widehat{x} c_{2}+x \widehat{\widetilde{x}} c_{4} \quad \rightsquigarrow \quad\{1,1,0,1,0,0\}=11 .
$$

With this coding the list of the 37 equations passing the conditions 1-3 of Definition 1 is given by

$$
\begin{aligned}
& \{5,\{1,0,1,0,0,0\}\},\{7,\{1,1,1,0,0,0\}\},\{10,\{0,1,0,1,0,0\}\},\{11,\{1,1,0,1,0,0\}\}, \\
& \{13,\{1,0,1,1,0,0\}\},\{14,\{0,1,1,1,0,0\}\},\{15,\{1,1,1,1,0,0\}\},\{21,\{1,0,1,0,1,0\}\}, \\
& \{23,\{1,1,1,0,1,0\}\},\{26,\{0,1,0,1,1,0\}\},\{27,\{1,1,0,1,1,0\}\},\{29,\{1,0,1,1,1,0\}\}, \\
& \{30,\{0,1,1,1,1,0\}\},\{31,\{1,1,1,1,1,0\}\},\{37,\{1,0,1,0,0,1\}\},\{39,\{1,1,1,0,0,1\}\}, \\
& \{42,\{0,1,0,1,0,1\}\},\{43,\{1,1,0,1,0,1\}\},\{45,\{1,0,1,1,0,1\}\},\{46,\{0,1,1,1,0,1\}\}, \\
& \{47,\{1,1,1,1,0,1\}\},\{48,\{0,0,0,0,1,1\}\},\{49,\{1,0,0,0,1,1\}\},\{50,\{0,1,0,0,1,1\}\}, \\
& \{51,\{1,1,0,0,1,1\}\},\{52,\{0,0,1,0,1,1\}\},\{53,\{1,0,1,0,1,1\}\},\{54,\{0,1,1,0,1,1\}\}, \\
& \{55,\{1,1,1,0,1,1\}\},\{56,\{0,0,0,1,1,1\}\},\{57,\{1,0,0,1,1,1\}\},\{58,\{0,1,0,1,1,1\}\}, \\
& \{59,\{1,1,0,1,1,1\}\},\{60,\{0,0,1,1,1,1\}\},\{61,\{1,0,1,1,1,1\}\},\{62,\{0,1,1,1,1,1\}\}, \\
& \{63,\{1,1,1,1,1,1\}\}
\end{aligned}
$$

Note that 5, 10 and 48 yield two-term equations.

In order to populate the consistency cube we need triplets of equations, leading in principle to $37^{3}=50653$ cases, fortunately this number can be reduced by applying symmetries.

\section{$2 \quad$ Symmetries}

For triplets of equations we use a list $\{X, Y, Z\}$ where $X$ is the decimal code for the bottom equation, $Y$ for the back equation and $Z$ for the left equation. The top, front and right equations are obtained by a coordinate shift and therefore there is no need to enumerate them separately. 
We will now look how some basic symmetries that do not change the CAC property operate on the codes. For easy reference we write here the triplet with $\iota$ but without lattice parameters:

$$
\begin{aligned}
& \iota_{1} x \widetilde{x} c_{1}+\iota_{2} \widetilde{x} \widehat{x} c_{2}+\iota_{3} \widehat{x} \widehat{\widetilde{x}} c_{3}+\iota_{4} x \widehat{\widetilde{x}} c_{4}+\iota_{5} x \widehat{x} c_{5}+\iota_{6} \widetilde{x} \widehat{\widetilde{x}} c_{6}=0, \\
& \iota_{1} x \widehat{x} c_{1}+\iota_{2} \widehat{x} \bar{x} c_{2}+\iota_{3} \bar{x} \bar{x} c_{3}+\iota_{4} x \overline{\widehat{x}} c_{4}+\iota_{5} x \bar{x} c_{5}+\iota_{6} \widehat{x} \bar{x} c_{6}=0 \\
& \iota_{1} x \bar{x} c_{1}+\iota_{2} \bar{x} \widetilde{x} c_{2}+\iota_{3} \widetilde{x} \bar{x} c_{3}+\iota_{4} x \overline{\widetilde{x}} c_{4}+\iota_{5} x \widetilde{x} c_{5}+\iota_{6} \bar{x} \bar{x} c_{6}=0 .
\end{aligned}
$$

\subsection{Rotation}

Let us first consider rotating the cube around the axis $(0,0,0)-(1,1,1)$, counterclockwise when looking from $(1,1,1)$. This moves the quadrilateral planes by $(n, m) \rightarrow(m, k) \rightarrow$ $(n, k) \rightarrow(n, m)$ and certainly does not change the consistency of the equations. Thus we rotate cyclically the shifts $\sim \rightarrow \rightarrow^{-} \rightarrow{ }^{\sim}$ accompanied with parameter change $p \rightarrow q \rightarrow r \rightarrow p$. Due to the cyclic convention in writing the triplet (3) it means that the LHS equations become

$$
\begin{aligned}
\text { back: } & Q_{12}(x, \widehat{x}, \bar{x}, \overline{\widehat{x}} ; q, r)=0, \\
\text { left: } & Q_{23}(x, \bar{x}, \widetilde{x}, \overline{\widetilde{x}} ; r, p)=0, \\
\text { bottom: } & Q_{31}(x, \widetilde{x}, \widehat{x}, \widehat{\widetilde{x}} ; p, q)=0
\end{aligned}
$$

This rotation does not change the roles of $c_{i}$ and therefore it corresponds to the rotation of triplet codes by

$$
\mathcal{R}\{X, Y, Z\}=\{Z, X, Y\}
$$

Clearly $\mathcal{R}^{3}=\mathbb{I}$. Using rotation symmetry we can reduce the number of equation triples to be analyzed, for example if we always rotate so that $X \leq Y, Z$ the remaining number of cases is 17575 (in practice we use a slightly different method).

\subsection{Tilde-hat reflection}

In addition to rotations we need to consider some reflections. Let us first take the reflection across the plane defined by points $(0,0,0)-(1,1,0)-(1,1,1),-(0,0,1)$, i.e. the tilde-hat

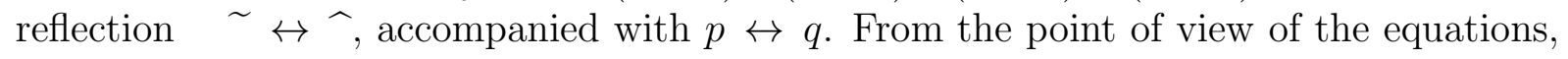
this rule is all we need to know to get another triplet of CAC equations. For the search process we need to know how this and other symmetries change the triplet codes of the equations, because we need to analyze only one triplet from the orbit of a triplet.

Applying tilde-hat reflection on (5) we get

$$
\begin{aligned}
& \iota_{1} x \widehat{x} c_{1}+\iota_{2} \widetilde{x} \widehat{x} c_{2}+\iota_{3} \widetilde{x} \widehat{\widetilde{x}} c_{3}+\iota_{4} x \widehat{\widetilde{x}} c_{4}+\iota_{5} x \widetilde{x} c_{5}+\iota_{6} \widehat{x} \widehat{\widetilde{x}} c_{6}=0, \\
& \iota_{1} x \widetilde{x} c_{1}+\iota_{2} \widetilde{x} \bar{x} c_{2}+\iota_{3} \bar{x} \bar{x} c_{3}+\iota_{4} x \overline{\widetilde{x}} c_{4}+\iota_{5} x \bar{x} c_{5}+\iota_{6} \widetilde{x} \overline{\widetilde{x}} c_{6}=0 \\
& \iota_{1} x \bar{x} c_{1}+\iota_{2} \bar{x} \widehat{x} c_{2}+\iota_{3} \widehat{x} \bar{x} c_{3}+\iota_{4} x \widehat{\widehat{x}} c_{4}+\iota_{5} x \widehat{x} c_{5}+\iota_{6} \bar{x} \bar{x} c_{6}=0 .
\end{aligned}
$$


Comparing this with (5) we find that the back and left equations get exchanged, and in addition the equations change by exchanging the $\iota$ or $c$ subscripts $1 \leftrightarrow 5$ and $3 \leftrightarrow 6$ (compare, e.g., (7c) with (5b)). Thus the tilde-hat reflection is given by the operator $\Theta$

$$
\Theta\{X, Y, Z\}=\{\theta X, \theta Z, \theta Y\}
$$

where the $\theta$ operator changes the binary codes by

$$
\theta\left\{\iota_{1}, \iota_{2}, \iota_{3}, \iota_{4}, \iota_{5}, \iota_{6}\right\}=\left\{\iota_{5}, \iota_{2}, \iota_{6}, \iota_{4}, \iota_{1}, \iota_{3}\right\}
$$

The above was for tilde-hat reflection, but we can of course also have hat-bar and bartilde reflections, these can be obtained using rotations: $\mathcal{R} \Theta \mathcal{R}^{2}$ and $\mathcal{R}^{2} \Theta \mathcal{R}$, respectively.

We have $\theta^{2}=\mathbb{I}$ and hence $\Theta^{2}=\mathbb{I}$, furthermore $\mathcal{R}^{2} \Theta=\Theta \mathcal{R},(\Theta \mathcal{R})^{2}=(\mathcal{R} \Theta)^{2}=\mathbb{I}$.

\subsection{Tilde reversal}

Another important reflection is the shift reversal. Let us consider the tilde reversal, which means reflection across the plane defined by the points $\left(\frac{1}{2}, 0,0\right)-\left(\frac{1}{2}, 1,0\right)-\left(\frac{1}{2}, 1,1\right)-$ $\left(\frac{1}{2}, 0,1\right)$, or alternatively, changing all tildes to down-tildes and then applying an overall tilde shift. This will exchange the back and front equations, which were related by shift, and this we can ignore. To see how it changes the bottom and left equation we apply the operation on (5) and obtain

$$
\begin{aligned}
& \iota_{1} x \widetilde{x} c_{1}+\iota_{2} x \widehat{\widetilde{x}} c_{2}+\iota_{3} \widehat{x} \widehat{\widetilde{x}} c_{3}+\iota_{4} \widetilde{x} \widehat{x} c_{4}+\iota_{5} \widetilde{x} \widehat{\widetilde{x}} c_{5}+\iota_{6} x \widehat{x} c_{6}=0, \\
& \iota_{1} x \widehat{x} c_{1}+\iota_{2} \widehat{x} \bar{x} c_{2}+\iota_{3} \bar{x} \bar{x} c_{3}+\iota_{4} x \overline{\widehat{x}} c_{4}+\iota_{5} x \bar{x} c_{5}+\iota_{6} \widehat{x} \bar{x} c_{6}=0 \\
& \iota_{1} \widetilde{x} \bar{x} c_{1}+\iota_{2} \overline{\widetilde{x}} x c_{2}+\iota_{3} x \bar{x} c_{3}+\iota_{4} \widetilde{x} \bar{x} c_{4}+\iota_{5} x \widetilde{x} c_{5}+\iota_{6} \bar{x} \overline{\widetilde{x}} c_{6}=0,
\end{aligned}
$$

Thus for the bottom equation we have $2 \leftrightarrow 4$ and $5 \leftrightarrow 6$, the back equation is unchanged, and for the left equation we have $1 \leftrightarrow 3$ and $2 \leftrightarrow 4$. We can write the action of the reversal operation $\mathcal{T}$ as

$$
\mathcal{T}\{X, Y, Z\}=\left\{T_{a} X, Y, T_{b} Z\right\}
$$

where

$$
T_{a}\left\{\iota_{1}, \iota_{2}, \iota_{3}, \iota_{4}, \iota_{5}, \iota_{6}\right\}=\left\{\iota_{1}, \iota_{4}, \iota_{3}, \iota_{2}, \iota_{6}, \iota_{5}\right\}
$$

and

$$
T_{b}\left\{\iota_{1}, \iota_{2}, \iota_{3}, \iota_{4}, \iota_{5}, \iota_{6}\right\}=\left\{\iota_{3}, \iota_{4}, \iota_{1}, \iota_{2}, \iota_{5}, \iota_{6}\right\} .
$$

We have $T_{a} T_{b}=T_{b} T_{a}, T_{a}^{2}=\mathbb{I}, T_{b}^{2}=\mathbb{I}$ and therefore $\mathcal{T}^{2}=\mathbb{I}$. The main relation between $T_{x}$ and $\theta$ is the conjugation $\theta T_{a} \theta=T_{b}$. We also have $\mathcal{R} \mathcal{T} \mathcal{R}^{2}=\Theta \mathcal{T} \Theta$, or alternatively $(\Theta \mathcal{R} \mathcal{T})^{2}=\mathbb{I}$ or $\mathcal{R} \mathcal{T} \mathcal{R}=\Theta \mathcal{T} \mathcal{R} \Theta$, also $(\mathcal{T} \Theta)^{4}=\mathbb{I}$

The above was for tilde-reversal, but we can of course also have hat-reversal and bar-reversal, these can be obtained considering: $\mathcal{R} \mathcal{T} \mathcal{R}^{2}$ and $\mathcal{R}^{2} \mathcal{T} \mathcal{R}$. 


\subsection{Inversion}

One important transformation that preserves affine linearity of the quad equation (up to overall factor) is the Moebius or affine linear transformation. Now that we are dealing with homogeneous equations only scaling $x \mapsto$ const. $\times x$ and inversion $x \mapsto 1 / x$ remain.

From (5) we can read that inversion $x \mapsto 1 / x$ implies $1 \leftrightarrow 3,2 \leftrightarrow 4$ and $5 \leftrightarrow 6$. Similar exchanges were also seen in tilde reversal above. Indeed one finds that $(\mathcal{R} \mathcal{T})^{3}$ implies $1 \leftrightarrow 3$ and $5 \leftrightarrow 6$, but not $2 \leftrightarrow 4$. Thus inversion would be an additional symmetry operation in cases where one of $c_{2}, c_{4}$ vanishes but not both. It will turn out that such an asymmetric possibility arises only with solutions in which $c_{2}$ and $c_{4}$ remain arbitrary functions, because then there is also solution in which one of $c_{2}, c_{4}$ vanishes. Such solutions are obtained by simple reductions and therefore we do not actually need inversion.

\subsection{Orbit of the transformation group}

By composing the basic operators of rotation $\mathcal{R}$ and two reflections $\mathcal{T}$ and $\Theta$ in various orders one can generate the orbit of a given initial triplet and for integrability we only need to check one representative of each orbit.

We have already mentioned some relations between the operators (and there may be others) and these can be used to eliminate some combinations as redundant. However, the full group generated by these three operations is probably rather large, but in our case we only need their representation when operating on three strings each containing six zeros or ones. It turns out that (after rotating the triplets into some canonical form) we only have orbits of length $1,2,4$ or 8 . Below we will give the orbits separately, if they have length $>1$.

\subsection{Gauge transformation}

Finally, we can change the coefficients in the equations by a gauge transformation of the form

$$
\begin{aligned}
x_{n, m, k} \rightarrow & a(p)^{n-n_{0}} b(q)^{m-m_{0}} c(r)^{k-k_{0}} \\
& \times A(p)^{\left(n-n_{0}\right)^{2}} B(q)^{\left(m-m_{0}\right)^{2}} C(r)^{\left(k-k_{0}\right)^{2}} \\
& \times \mathcal{A}(p, q)^{\left(n-n_{0}\right)\left(m-m_{0}\right)} \mathcal{B}(q, r)^{\left(m-m_{0}\right)\left(k-k_{0}\right)} \mathcal{C}(r, p)^{\left(k-k_{0}\right)\left(n-n_{0}\right)} x_{n, m, k},
\end{aligned}
$$

where we have also included terms with quadratic exponent. However, there are strong conditions on the allowed values for $a, b, c, A, B, C, \mathcal{A}, \mathcal{B}, \mathcal{C}$ because the transformation must not break the uniformity of the $3 \mathrm{D}$ lattice, i.e., the translation invariance of the quad equation. (Note that for non-homogeneous equations the gauge freedom would be considerably smaller, perhaps only leaving sign changes.)

In order to check the uniformity easily we have introduced a fixed point $n_{0}, m_{0}, k_{0}$, and when the gauge transformation is applied to a given equation the result cannot depend 
on the fixed point, except possibly through an overall multiplier. The restrictions this imposes on the parameters of the gauge transformation will depend on the particular triplet of equations.

We will use gauge transformations to simplify the final result, for example by eliminating some free coefficient(s) from the equation(s), when it simplifies the system. However, even if we can eliminate some free function $c_{i}$ this way, the mere possibility that such a function exists is by itself important for classification.

\section{The search}

Solving the equations that result from the CAC conditions cannot be fully automatized and therefore we cannot separately solve each one of the tentative number of 17575 cases (obtained with $X \leq Y, Z$ ). For this reason further computer scanning was done by checking whether the equations obtained from the CAC condition contain any that are monomials of the $c_{j}$. Since $c_{j}$ are all nonzero the monomial equation cannot be satisfied and therefore that equation triplet can be omitted. This scan turned out to effective, and it was possible to analyze the remaining list of triplets (one representative per orbit).

For the triplet codes remaining after scanning we studied the equations generated by CAC until we got into a contradiction with $c_{i} \neq 0$, or one of the equations in the triplet factorized, or in the positive case, all equations were solved. The search and the results obtained are given in Appendix A. As discussed there, we grouped the set of codes remaining after rough scanning into blocks, and only one representative of an orbit was tested. We have freely used gauge transformations (11) and rotations (6) to present the triplet equations in a nice form, for example so that the "side equations" are similar.

In the next section we group and classify the result of Appendix A.

\section{Classification}

The primary classification of the results is by whether or not the triplet of equations can contain free functions of two variables $c_{i}$. The gauge transformation (11) can sometimes be used to eliminate such a function but what is relevant is whether such functions can appear (other than as overall factors).

It is also useful to observe the appearance of the $c_{2}, c_{4}$ pair, that is, terms of the type

$\widetilde{x} \widehat{x}+x \widehat{\widetilde{x}}$. This is useful since the pair does not get mixed up with other terms in any of the reflections.

The results we have obtained can be grouped into four sets, each set having a highest equation from which the remaining equations are obtained by reductions, possibly accompanied by rotations and reflections. 


\section{$4.1\{63,10,10\}$}

For the first group the most general triplet is

$$
\begin{aligned}
x \widetilde{x} c_{1}(p, q)+\widetilde{x} \widehat{x} c_{2}(p, q)+\widehat{x} \widehat{\widetilde{x}} c_{3}(p, q)+x \widehat{\widetilde{x}} c_{4}(p, q)+x \widehat{x} c_{5}(p, q)+\widetilde{x} \widehat{\widetilde{x}} c_{6}(p, q) & =0, \\
\widehat{x} \bar{x}-x \widehat{\widehat{x}} & =0 \\
\bar{x} \widetilde{x}-x \overline{\widetilde{x}} & =0 .
\end{aligned}
$$

Thus the bottom equation is a completely free homogeneous quadratic equation while the side equations are simple. This is Equation (33) in Appendix A.1. The triply shifted $x$ is given by

$$
\overline{\widehat{\widetilde{x}}}=-\frac{c_{5}(p, q) x \bar{x} \widehat{x}+c_{1}(p, q) x \widetilde{x} \bar{x}+c_{2}(p, q) \widetilde{x} \widehat{x} \bar{x}}{c_{4}(p, q) x^{2}+c_{6}(p, q) x \widetilde{x}+c_{3}(p, q) x \widehat{x}} .
$$

We see that this equation has the tetrahedron property if $c_{2}=c_{4}=0$. The organization of the 36 sub-cases, obtained by setting some $c_{j}=0$, into orbits is given in Appendix A.2. The simplest equation in this category is (32) in Appendix A.1.

For $\{63,10,10\}$ one cannot change the back or left equations by gauge, but as the number of terms in the bottom equation decreases there is more freedom in the side equations. This is illustrated by a CAC result in the $\{58,10,10\}$ category:

$$
\begin{aligned}
\widetilde{x} \widehat{x} c_{2}(p, q)+x \widehat{\widetilde{x}} c_{4}(p, q)+x \widehat{x} c_{5}(p, q)+\widetilde{x} \widehat{\widetilde{x}} c_{6}(p, q) & =0, \\
c_{2}(q, r) \bar{x} \widehat{x}+c_{4}(q, r) x \widehat{\widehat{x}} & =0, \\
\widetilde{x} \bar{x}-x \overline{\widetilde{x}} & =0 .
\end{aligned}
$$

It has two free functions in the back equation. However it is transformed into a sub-case of (12) by the gauge transformation

$$
x_{n, m, k} \rightarrow\left(-c_{2}(q, r) / c_{4}(q, r)\right)^{\left(m-m_{0}\right)\left(k-k_{0}\right)} x_{n, m, k} .
$$

The possibility of extra free functions that can be gauged away is irrelevant for MDC, but they will have information value in the analysis of BTs in Section 5.4.

\section{$4.2 \quad\{10,58,15\}$}

This triplet is Equation (34) in Appendix A.2. Two of the equations have four free functions each

$$
\begin{aligned}
\widehat{x} \widetilde{x}-x \widehat{\widetilde{x}} & =0 \\
x \bar{x} c_{5}(q, r)+x \overline{\widehat{x}} c_{4}(q, r)+\bar{x} \widehat{x} c_{2}(q, r)+\overline{\widehat{x}} \widehat{x} c_{6}(q, r) & =0 \\
x \bar{x} c_{1}(r, p)+x \bar{x} c_{4}(r, p)+\bar{x} \widetilde{x} c_{2}(r, p)+\overline{\widetilde{x}} \widetilde{x} c_{3}(r, p) & =0 .
\end{aligned}
$$

Note that the last two equations are not connected by a cyclic variable change but by just tilde-hat exchange (and cyclic parameter change). The triply shifted quantity is given by

$$
\overline{\widehat{x}}=\bar{x} \frac{\left(c_{1}(r, p) x+c_{2}(r, p) \widetilde{x}\right)\left(c_{5}(q, r) x+c_{2}(q, r) \widehat{x}\right)}{\left(c_{4}(r, p) x+c_{3}(r, p) \widetilde{x}\right)\left(c_{4}(q, r) x+c_{6}(q, r) \widehat{x}\right)} .
$$


The sub-cases of (14) are listed in Appendix A.3 and are obtainable by setting some $c_{j}=0$. For each of the four term equations there are 7 acceptable sub-cases but one of them leads to the $\{63,10,10\}$ category, therefore there are 36 cases in this category. Missing $c_{j}$ terms sometimes allow a more general bottom equation, but that freedom can be gauged away. TET is possible only if $c_{2}(r, p)=c_{4}(r, p)=c_{5}(q, r)=c_{6}(q, r)=0$ or if $c_{1}(r, p)=c_{3}(r, p)=c_{2}(q, r)=c_{4}(q, r)=0$, but both reduce $(14)$ to the $\{\mathrm{X}, 10,10\}$ category.

\section{$4.3 \quad\{53,15,58\}$}

Another chain of triplets that can have free functions is obtained starting with the highest equation $\{\mathbf{5 3}, \mathbf{1 5}, \mathbf{5 8}\}$ or Equation (41) of Appendix A.5. After a gauge transformation the result can be written as

$$
\begin{aligned}
c_{6}(p, q) \widehat{\widetilde{x}} \widetilde{x}+c_{3}(p, q) \widehat{x} \widehat{\widetilde{x}}+c_{1}(p, q) x \widetilde{x}+c_{5}(p, q) x \widehat{x} & =0 \\
r(x \widehat{x}+\bar{x} \bar{x})+x \bar{x}+\bar{x} \widehat{x} & =0 \\
r(x \widetilde{x}+\bar{x} \bar{x})+x \overline{\widetilde{x}}+\bar{x} \widetilde{x} & =0 .
\end{aligned}
$$

The triply shifted variable is

$$
\overline{\widehat{x}}=-\bar{x} \frac{c_{1}(p, q) \widetilde{x}+c_{5}(p, q) \widehat{x}}{c_{6}(p, q) \widetilde{x}+c_{3}(p, q) \widehat{x}},
$$

and clearly has the TET property. The sub-cases obtained by setting some $c_{i}=0$ change the first number of the triplet code and are listed in Appendix A.5. Other types of reductions are given in Figure 2, they are due to limits and are explained below.

1) $\{53,11,26\}$ is obtained from $\{53,15,58\}$ by scaling $\bar{x} \rightarrow r \bar{x}$ and then taking the leading term as $r \rightarrow 0$.

2) $\{53,05,48\}$ is obtained from $\{53,15,58\}$ by taking the leading term as $r \rightarrow \infty$.

3) If $r=0$ the triplet (15) reduces to a sub-case $\{53,10,10\}$ of (12).

4) If in $\{21,05,48\}$ we take $c_{5}=0$, we get $\{05,05,48\}$ which is rotated reflected (49).

\section{$4.4\{63,48,05\}$}

Here the highest equation is Equation (51) of Appendix A.8:

$$
\begin{aligned}
c_{1}(p, q)\left(x \widetilde{x}-\sigma_{1} \sigma_{3} \widehat{x} \widehat{\widetilde{x}}\right)+c_{5}(p, q)\left(x \widehat{x}-\sigma_{1} \sigma_{2} \widehat{\widetilde{x}} \widetilde{x}\right)+c_{4}(p, q)\left(\widehat{x} \widetilde{x}-\sigma_{1} x \widehat{\widetilde{x}}\right) & =0, \quad(16 \mathrm{a}) \\
x \bar{x}-\sigma_{3} \overline{\widehat{x}} \widehat{x} & =0, \quad(16 \mathrm{~b}) \\
x \bar{x}-\sigma_{2} \overline{\widetilde{x}} \widetilde{x} & =0 . \quad(16 \mathrm{c})
\end{aligned}
$$




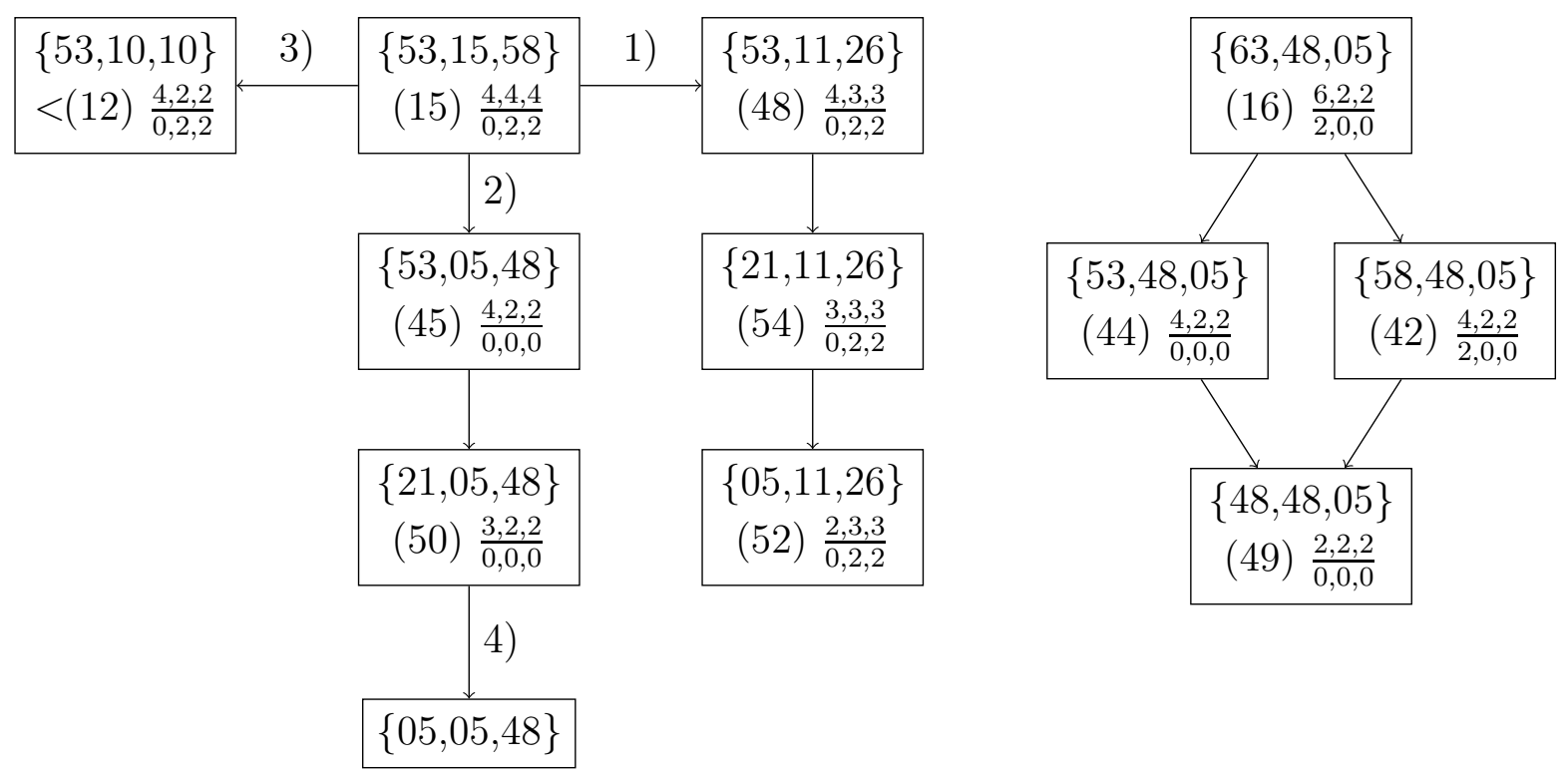

Figure 2: On the left some sub-case relations from $\{53,15,58\}$, on the right the reductions from $\{63,48,05\}$. Each box gives the code of the triplet and under it on the left its equation number. The ratio on the right gives above the number of terms and below the number of $c_{2}, c_{4}$ terms.

The sign $\sigma_{1}$ cannot be eliminated by gauge.

$$
\overline{\widehat{x}}=\frac{x \bar{x}\left(c_{1}(p, q) \sigma_{3} \widehat{x}+c_{4}(p, q) x+c_{5}(p, q) \sigma_{2} \widetilde{x}\right)}{\sigma_{1} \sigma_{2} \sigma_{3}\left(c_{1}(p, q) x \widetilde{x}+c_{4}(p, q) \widehat{x} \widetilde{x}+c_{5}(p, q) x \widehat{x}\right)} .
$$

It has TET if $c_{4}=0$, which has code $\{48,48,05\}$. The diagram on the RHS of Figure 2 describes the reductions of (16), they are all by setting some $c_{i}=0$.

\section{$4.5\{63,63,63\}-2$, a triplet without free functions}

The highest level triplet of this set has code $\{63,63,63\}-2$, it is Equation (59) in Appendix. It is in fact $Q 3(\delta=0)$ of the ABS list [2]:

$$
\begin{aligned}
\left(q^{2}-p^{2}\right)(x \widehat{\widetilde{x}}+\widehat{x} \widetilde{x})-p\left(q^{2}-1\right)(x \widehat{x}+\widehat{\widetilde{x}} \widetilde{x})+\left(p^{2}-1\right) q(x \widetilde{x}+\widehat{x} \widehat{\widetilde{x}}) & =0, \\
\left(r^{2}-q^{2}\right)(x \overline{\widehat{x}}+\bar{x} \widehat{x})-q\left(r^{2}-1\right)(x \bar{x}+\overline{\widehat{x}} \widehat{x})+\left(q^{2}-1\right) r(x \widehat{x}+\bar{x} \bar{x}) & =0, \\
\left(p^{2}-r^{2}\right)(x \overline{\widetilde{x}}+\widetilde{x} \bar{x})-r\left(p^{2}-1\right)(x \widetilde{x}+\overline{\widetilde{x}} \bar{x})+\left(r^{2}-1\right) p(x \bar{x}+\widetilde{x} \overline{\widetilde{x}}) & =0 .
\end{aligned}
$$

The triply shifted variable is

$$
\overline{\widehat{x}}=\frac{\bar{x} \widehat{x} p\left(q^{2}-r^{2}\right)+\bar{x} \widetilde{x} q\left(r^{2}-p^{2}\right)+\widehat{x} \widetilde{x} r\left(p^{2}-q^{2}\right)}{\bar{x} r\left(q^{2}-p^{2}\right)+\widehat{x} q\left(p^{2}-r^{2}\right)+\widetilde{x} p\left(r^{2}-q^{2}\right)} .
$$

Furthermore, all other triplets that cannot have free functions are obtained by some limit from this equation. Figure 3 describes the chain of limits. From each orbit one element 
is mentioned, its code, equation number, as well as the number of terms in each equation and the number of $\widetilde{x} \widehat{x}+x \widehat{\widetilde{x}}$-type terms.

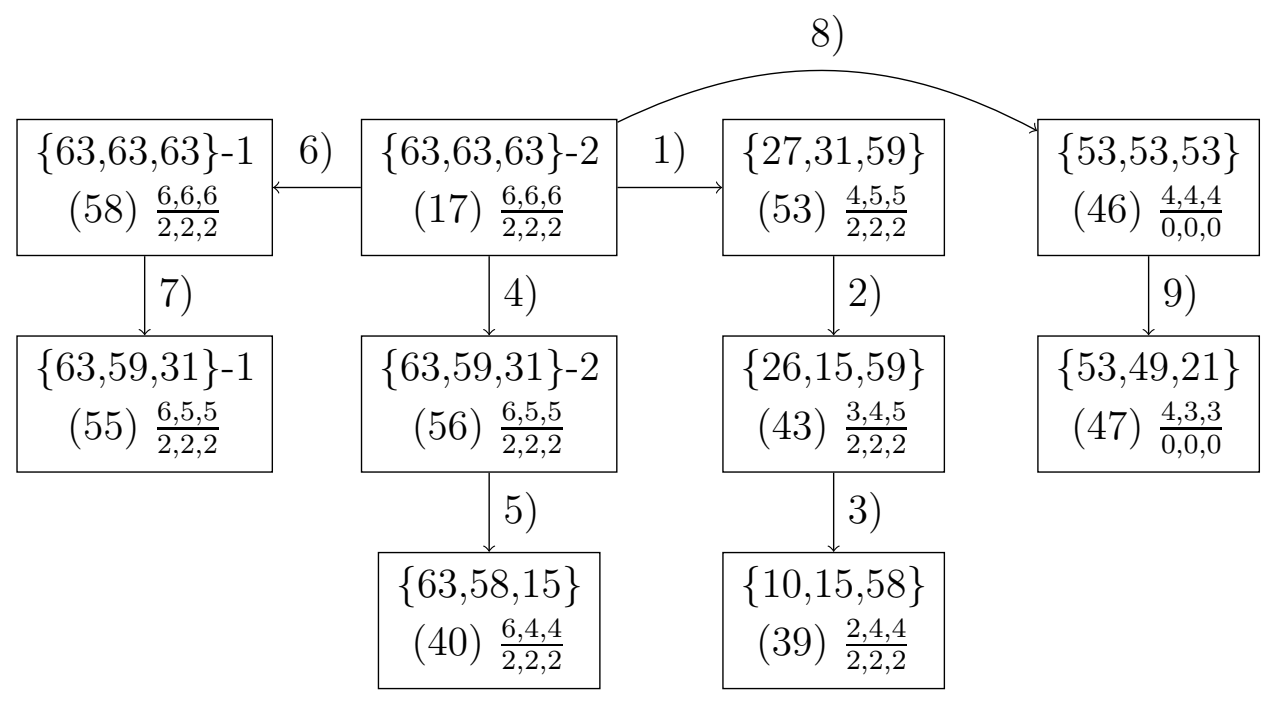

Figure 3: Sub-case relations, same notation as in Figure 2. The starting Equation $\{63,63,63\}-2(17)$ is $\mathrm{Q} 3(\delta=0),\{63,63,63\}-1(58)$ is $\mathrm{Q} 1(\delta=0)$ and $\{53,53,53\}(46)$ is $\mathrm{H} 3(\delta=0)$. Every reduction simplifies the triplet of equations and eventually we reach equations that can contain free functions $c_{j}$.

The limits given in Figure 3 are obtained as follows:

1) In (17) scale $\widetilde{x} \rightarrow-\widetilde{x} / p, \widehat{x} \rightarrow-q \widehat{x}$ and change $r \rightarrow-1 / r$ and then take the leading terms as $p \rightarrow \infty, q \rightarrow 0$.

2) In (53) scale $\widehat{x} \rightarrow q \widehat{x}$ and then take the leading term as $q \rightarrow \infty$.

3) In (43) scale $\widetilde{x} \rightarrow p \widetilde{x}$ and then take the leading term as $p \rightarrow \infty$. In addition replace $r \mapsto 1 / r$ and change signs by gauge.

4) In (17) scale $\bar{x} \rightarrow \bar{x} / r$ and then take the leading term as $r \rightarrow \infty$. Also change signs of $p, q$.

5) In (56) scale $\bar{x} \rightarrow r \bar{x}$ and then take the leading term as $r \rightarrow \infty$.

6) In (17) let $p \mapsto 1+\epsilon p, q \mapsto 1+\epsilon q, r \mapsto 1+\epsilon r$ and take the leading term as $\epsilon \rightarrow 0$.

7) In (58) scale $\bar{x} \rightarrow \bar{x} / r, \widetilde{x} \rightarrow-\widetilde{x}, \widehat{x} \rightarrow-\widehat{x}$ and then take leading term in $r \rightarrow \infty$.

8) In (17) take the leading term (linear) as $p, q, r \rightarrow 0$.

9) In (46) scale $\bar{x} \rightarrow r \bar{x}$ and then take the leading terms as $r \rightarrow 0$. 
In this way all equations to which free functions of two variables cannot be introduced by a gauge are reductions of $Q 3(\delta=0)(17)$.

It should be noted, however, that in Figure 3 the bottom box $\{10,15,58\}$ (39) is an equation that does allow free functions, and this happens also on further reductions from the other bottom boxes: From $\{63,58,15\}$ (40) the limit $p, q \rightarrow 0$ leads to $\{53,10,10\}$ while $p, q \rightarrow \infty$ leads to $\{53,48,05\}(44)$; from $\{10,15,58\}$ (39) the limit $r \rightarrow$ leads to $\{10,05,10\}$ while $r \rightarrow \infty$ leads to $\{10,10,48\}$, which are in the same orbit; from $\{53,49,21\}$ (47) the limit $p, q \rightarrow \infty$ leads to $\{53,48,05\}$ (44). All of these further limits yield equations which allow free functions. It should be noted also that some potential limits actually lead to factorizable equations, for example if in $\{63,59,31\}-1$ (55) one takes $p, q \rightarrow 0$ or if in $\{63,58,15\}$ (40) one takes $p \mapsto 1+\epsilon p, q \mapsto 1+\epsilon q, r \mapsto 1+\epsilon r$ and then take the leading term as $\epsilon \rightarrow 0$.

We also note that (17) still allows gauge transformations of the form

$$
x_{n, m, k} \rightarrow a(p)^{n-n_{0}} b(q)^{m-m_{0}} c(r)^{k-k_{0}} \sigma_{1}^{\left(n-n_{0}\right)^{2}} \sigma_{2}^{\left(m-m_{0}\right)^{2}} \sigma_{3}^{\left(k-k_{0}\right)^{2}}
$$

where $\sigma_{j}^{2}=1$. This changes the bottom equation into

$[x \widehat{\widetilde{x}}+\widehat{x} \widetilde{x}]\left(q^{2}-p^{2}\right)-\left[x \widehat{x} a(p)^{-1}+\widehat{\widetilde{x}} \widetilde{x} a(p)\right] \sigma_{1} p\left(q^{2}-1\right)+\left[x \widetilde{x} b(q)^{-1}+\widehat{x} \widehat{\widetilde{x}} b(q)\right] \sigma_{2} q\left(p^{2}-1\right)=0$

and corresponding results hold for the other equations.

\subsection{Comparison with Boll's results}

As was mentioned before, Boll has done a classification of triplets of CAC equations in $[3,4,5]$. His setup was both more general (allowing flips between opposing sides and not restricting to homogeneous quadratic equations) and less general (by requiring the tetrahedron property). In [3] the results were collected into Theorems 3.4-3.11. Restricting those equations to quadratic irreducible equations without flips implies some restrictions on the parameters, and yield the following:

1. For (3.6),(3.4) we must take $\delta=0, \epsilon=0$, then it gives (46), i.e. H3( $\delta=0)$. If $\gamma=0$ we get $(44)$

2. For $(3.10),(3.7)$ we must take $\delta=0$, then it gives $\{47,63,62\}$, which is reflected rotated (56). If furthermore $\epsilon=0$ we get rotated (40).

3. For (3.14),(3.11) we must take $\delta=0, \epsilon=0$, then it gives (15). If furthermore we take $\gamma=0$ we get (44). (Also for $\gamma^{2}=1$ there are no flips but then some equations factorize.)

4. In (3.19-20) one additional parameter dependence is missing in $B$, this is corrected in [4]. In the corrected form it is necessary to take $\delta_{3}=0$, which yields reflected (53). If after this we take $\delta_{1}=0$ or $\delta_{2}=0$ we get a reflected forms of (43) and if both coefficients vanish we get (39). 
5. For (3.21-22) (of [3], not [4]) we must take $\delta_{1}=0$ or change $\delta x_{1} x_{2}$ into $\delta x_{1} x$, which is again a special case of (15). If $\alpha^{2}=1$ some equations factorize.

6. For (3.29-30) we must take $\delta_{2}=0$, then it gives reflected rotated (47). If furthermore $\delta_{1}=0$ we get rotated (44).

7. For (3.31-32) we must take $\delta_{1}=\delta_{2}=0$, then it gives rotated (45). In that case all coefficients in $B$ of (3.31) can in fact be free. If in (3.31) we take $\alpha=0$ we get rotated (49).

8. For (3.33-34) we must take $\delta_{1}=0$, then it gives reflected (48). In $B$ all coefficients can be free.

9. For (3.41-42) we must take $\delta_{1}=0$, then it corresponds to $\{42,21,14\}$, a rotated form of which appears in the orbit of $(54)$. Next if $\delta_{3}=0$ we get rotated reflected (52) and if $\delta_{2}=0$ we get subcase $\{53,10,10\}$ of $(12)$.

10. For (3.43-44) we must take $\delta_{1}=\delta_{2}=0$, then it becomes rotated form of (50). (Note that in the formula for $C$ the first variable must be $x_{13}$, not $x_{12}$.)

Thus all homogeneous quadratic shift invariant equations in Boll's classification [3] are also included in our classification. However, there is one result we could not identify among Boll's results, namely (55), which is related to Q1 the same way that (56) is related to Q3, and (46) is related to H3.

\subsection{Triplets with two term equations}

As another selection of our results we would like to collect here the simplest CAC triplets, those for which all equations have just two terms. Below on the LHS they are given in the general form after all equations from the CAC condition have been solved, and then on the RHS we present the simplest form obtainable by a gauge transform.

- $\{10,10,10\}$

$$
\left\{\begin{array} { l } 
{ c _ { 2 } ( p , q ) \widetilde { x } \widehat { x } + c _ { 4 } ( p , q ) x = 0 , } \\
{ c _ { 2 } ( q , r ) \widehat { x } \overline { x } + c _ { 4 } ( q , r ) x = 0 , } \\
{ c _ { 2 } ( r , p ) \overline { x } \widetilde { x } + c _ { 4 } ( r , p ) x = 0 , }
\end{array} \quad \rightarrow \quad \left\{\begin{array}{l}
\widetilde{x} \widehat{x}-x \widehat{\widetilde{x}}=0 \\
\widehat{x} \bar{x}-x \overline{\widehat{x}}=0 \\
\bar{x} \widetilde{x}-x \overline{\widetilde{x}}=0
\end{array}\right.\right.
$$

- $\{\mathbf{5 , 1 0 , 1 0}\}$ or $\{48,10,10\}$ by tilde-hat reflection

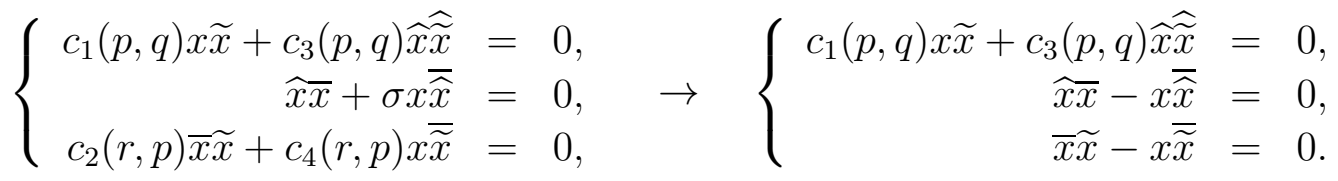


- $\{10,48,5\}$

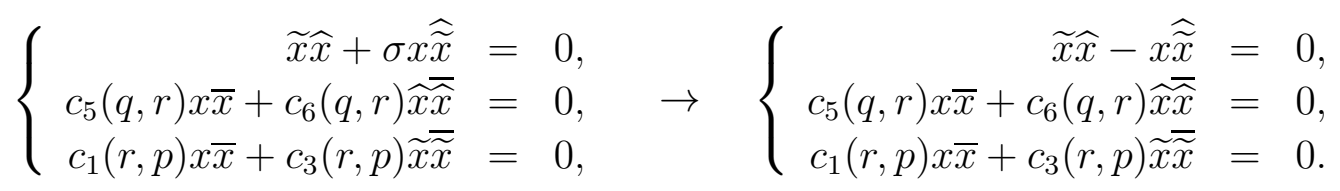

- $\{5,5,48\}$ and $\{48,5,48\}$ by tilde-hat reflection

$$
\left\{\begin{array} { r l } 
{ c _ { 1 } ( p , q ) x \widetilde { x } + c _ { 3 } ( p , q ) \widehat { x } \widehat { \widetilde { x } } } & { = 0 , } \\
{ c ( r ) x \widehat { x } + \overline { x } \widehat { x } } & { = 0 , } \\
{ \sigma c ( r ) x \widetilde { x } + \overline { x } \overline { x } } & { = 0 , }
\end{array} \rightarrow \left\{\begin{array}{rl}
c_{1}(p, q) x \widetilde{x}+c_{3}(p, q) \widehat{x} \widehat{\widetilde{x}}= & 0, \\
x \widehat{x}-\bar{x} \widehat{x}=0, & x \widetilde{x}-\bar{x} \overline{\widetilde{x}}=0 .
\end{array}\right.\right.
$$

These cases are easily distinguished by the number of $c_{2}, c_{4}$ terms, which have also been rotated into a particular position. These triplets can be obtained from several results listed in the Appendix by various limits.

\section{Summary:}

The triplets that were found using CAC can be divided into two groups: 1) Those that cannot have free functions of two lattice parameters are all reductions of $Q 3(\delta=0)$ of the ABS list [2]. 2) The triplets that can have free functions of two lattice parameters can be organized into 4 sets, with the highest triplets of the set being $\{63,10,10\},\{53,15,58\}$, $\{63,48,05\}$, or $\{10,58,15\}$.

\section{Bäcklund transformations and Lax pairs}

The equations that were found using $\mathrm{CAC}$ will next be studied from the point of view of their Bäcklund transformations and Lax pairs.

\subsection{General setup}

Let us fix the equations and some concepts. We have six equations, which we can arrange as follows:

$$
\begin{gathered}
Q_{12}(y, \widetilde{y}, \widehat{y}, \widehat{\widetilde{y}} ; p, q)=0 \\
Q_{23}(x, \widehat{x}, y, \widehat{y} ; q, r)=0, \quad Q_{23}(\widetilde{x}, \widehat{\widetilde{x}}, \widetilde{y}, \widehat{\widetilde{y}} ; q, r)=0 \\
Q_{31}(x, y, \widetilde{x}, \widetilde{y} ; r, p)=0, \quad Q_{31}(\widehat{x}, \widehat{y}, \widehat{\widetilde{x}}, \widehat{\widetilde{y}} ; r, p)=0 \\
Q_{12}(x, \widetilde{x}, \widehat{x}, \widehat{\widetilde{x}} ; p, q)=0 .
\end{gathered}
$$

This is the same set as (3), except that we have named $\bar{x}=y$, which has no effect on the algebra. We see that the top equation (23a) depends only on the $y$ variables, the bottom equation (23d) only on the $x$ variables, while the side equations (23b),(23c) depend on two $x$ and on two $y$ variables. 
Definition 2 (Bäcklund transform of equations (eqBT)) Consider equations (23b) and (23c) and solve for $\widehat{y}$ and $\widetilde{y}$ from their LHS. Then from the RHS of these equations we can solve for $\widehat{\widetilde{y}}$ in two ways. If these two results are automatically equal, we say that the eqBT is trivial, i.e., it fails. If the results are different we factorize the numerator of their difference and take all factors that only depend on $x, \widetilde{x}, \widehat{x}, \widehat{\widetilde{x}}$. If there is a unique factor that is also an acceptable equation the we say that the eqBT is strong and the generated equation is called $Q_{12}(23 \mathrm{~d})$. If there are several acceptable factors we say that the eqBT is weak. The same process can be done by eliminating the $x$ functions, leading (possibly) to an equation in $y$.

Note that if the system (23) has the CAC property then the above mentioned two results for $\widehat{\widetilde{y}}$ will be the same either automatically or modulo $Q_{12}$.

Definition 3 (Bäcklund transform of solutions (solBT)) Suppose $x$ is a solution of (23d). If we can solve for $y$ from (23b) and (23c) and that solution solves (23a) then we have a BT transforming solutions. If $x=y$ then the transformation is trivial.

Since we are only interested in the equations and their relationships we only use eqBT as described in Definition 2.

Before doing any computations we can already make some statements about the triplets that we have found. The starting setup was that all coefficients in the triplet of equations were free functions depending on the two lattice parameters associated with that quadrilateral, and then from the CAC we got conditions due to which some twovariable functions were fixed. However, we have seen that sometimes even if a triplet is CAC it may still contain arbitrary functions $c_{i}$ (other than overall factors).

If we now look at this from the point of view of eqBT, it is clear that an equation with a free function cannot be generated by an eqBT composed of the remaining pair of equations, because of different dependence on lattice parameters. For example, when the side equations only contain $c_{j}(q, r)$ and $c_{j}(r, p)$ they cannot be used to construct arbitrary free $c_{j}(p, q)$. Furthermore, since gauge transformations have no effect on the eqBT, it turns out that the important property is the possibility of free functions (other than overall multipliers). For example for (32) BT produces nothing and this can be predicted from the fact that by gauge freedom each equation can have free functions as shown in (31). In the case of the triplet (13) the bottom and back equations may contain free functions and therefore a eqBT can at most produce the back equation.

In comparing eqBT and $\mathrm{CAC}$ we note that for $\mathrm{CAC}$ there is no fundamental difference in the roles of the equations, while for eqBT the middle equations (23b), (23c) form the transformation using which we should be able to generate top and bottom equations. In a closer analysis we observe that for CAC and eqBT the computations start the same way: If we take $x, \widetilde{x}, \widehat{x}, y$ as initial values we can solve for $\widehat{y}$ in terms of $x, \widehat{x}, y$ from the back equation and $\widetilde{y}$ in terms of $x, \widetilde{x}, y$ from the left equation. Then solving for $\widehat{\widetilde{y}}$ from the right equation we have three equations remaining, bottom equation, which depends on $x, \widetilde{x}, \widehat{x}, \widehat{\widetilde{x}}$, and top and front equations which now depend on $x, \widetilde{x}, \widehat{x}, \widehat{\widetilde{x}}, y$ At this point the $\mathrm{CAC}$ and $\mathrm{BT}$ methods diverge. 
- In the next step for CAC we would solve for $\widehat{\widetilde{x}}$ from the bottom equation and when it is substituted into the two remaining equations they should vanish identically.

- In eqBT we are only working with $Q_{23}$ and $Q_{31}$, and therefore only have the front equation remaining. It should now factorize with one factor being the bottom equation, the other factor possibly containing $y$.

In practice the methods differ when the front equation vanishes before substituting $\widehat{\widetilde{x}}$, in which case we clearly cannot generate the bottom equation.

We note also that in (23) the roles of $x$ and $y$ are symmetric and therefore the same arguments can be used to generate the top equation. Furthermore, if we instead rename $\widehat{x}=y$ in (3) we get a set from which the above procedure can be used, mutatis mutandis, for generating left or right equations, and if we take $\widetilde{x}=y$ we can generate back and front equations.

\subsection{Computations for eqBT}

In order to further analyze the eqBT, it is useful to develop the formulae to some extent without specifying the form of the $Q$-polynomials. For this purpose it is often useful to isolate one of the variables of $Q$, but since $Q$ is multilinear this is easy. We can write, for example,

$$
Q_{12}(x, \widetilde{x}, \widehat{x}, \widehat{\widetilde{x}} ; p, q)=Q_{12}(x, \widetilde{x}, \widehat{x}, \bullet ; p, q) \widehat{\widetilde{x}}+Q_{12}(x, \widetilde{x}, \widehat{x}, 0 ; p, q)
$$

where

$$
Q_{12}(x, \widetilde{x}, \widehat{x}, \bullet ; p, q):=\frac{\partial}{\partial \widehat{\widetilde{x}}} Q_{12}(x, \widetilde{x}, \widehat{x}, \widehat{\widetilde{x}} ; p, q)=Q_{12}(x, \widetilde{x}, \widehat{x}, 1 ; p, q)-Q_{12}(x, \widetilde{x}, \widehat{x}, 0 ; p, q)
$$

Using this notation we can solve the LHS equations of (23b), (23c),

$$
\begin{aligned}
\widehat{y} & =-\frac{Q_{23}(x, \widehat{x}, y, 0 ; q, r)}{Q_{23}(x, \widehat{x}, y, \bullet ; q, r)}, \\
\widetilde{y} & =-\frac{Q_{31}(x, y, \widetilde{x}, 0 ; r, p)}{Q_{31}(x, y, \widetilde{x}, \bullet ; r, p)} .
\end{aligned}
$$

and then from the RHS equations we can solve

$$
\begin{aligned}
\widehat{\widetilde{y}}_{23} & =-\frac{Q_{23}(\widetilde{x}, \widehat{\widetilde{x}}, \widetilde{y}, 0 ; q, r)}{Q_{23}(\widetilde{x}, \widehat{\widetilde{x}}, \widetilde{y}, \bullet ; q, r)} \\
& =\frac{Q_{23}(\widetilde{x}, \widehat{\widetilde{x}}, \bullet, 0 ; q, r) Q_{31}(x, y, \widetilde{x}, 0 ; r, p)-Q_{23}(\widetilde{x}, \widehat{\widetilde{x}}, 0,0 ; q, r) Q_{31}(x, y, \widetilde{x}, \bullet ; r, p)}{Q_{23}(\widetilde{x}, \widehat{\widetilde{x}}, \bullet, \bullet ; q, r) Q_{31}(x, y, \widetilde{x}, 0 ; r, p)-Q_{23}(\widetilde{x}, \widehat{\widetilde{x}}, 0, \bullet ; q, r) Q_{31}(x, y, \widetilde{x}, \bullet ; r, p)} \\
\widehat{\widetilde{y}}_{31} & =-\frac{Q_{31}(\widehat{x}, \widehat{y}, \widehat{\widetilde{x}}, 0 ; r, p)}{Q_{31}(\widehat{x}, \widehat{y}, \widehat{\widetilde{x}}, \bullet ; r, p)} \\
& =\frac{Q_{31}(\widehat{x}, \bullet, \widehat{\widetilde{x}}, 0 ; r, p) Q_{23}(x, \widehat{x}, y, 0 ; q, r)-Q_{31}(\widehat{x}, 0, \widehat{\widetilde{x}}, 0 ; r, p) Q_{23}(x, \widehat{x}, y, \bullet ; q, r)}{Q_{31}(\widehat{x}, \bullet, \widehat{\widetilde{x}}, \bullet ; r, p) Q_{23}(x, \widehat{x}, y, 0 ; q, r)-Q_{31}(\widehat{x}, 0, \widehat{\widetilde{x}}, \bullet ; r, p) Q_{23}(x, \widehat{x}, y, \bullet ; q, r)}
\end{aligned}
$$


Let us furthermore expand these in $y$ since it cannot appear in final equation. We have

$$
\begin{aligned}
& \widehat{\widetilde{y}}_{23}=-\frac{W_{1}(x, \widetilde{x}, \widehat{\widetilde{x}}) y+W_{2}(x, \widetilde{x}, \widehat{\widetilde{x}})}{W_{3}(x, \widetilde{x}, \widehat{\widetilde{x}}) y+W_{4}(x, \widetilde{x}, \widehat{\widetilde{x}})} \\
& \widehat{\widetilde{y}}_{31}=-\frac{Z_{1}(x, \widehat{x}, \widehat{\widetilde{x}}) y+Z_{2}(x, \widehat{x}, \widehat{\widetilde{x}})}{Z_{3}(x, \widehat{x}, \widehat{\widetilde{x}}) y+Z_{4}(x, \widehat{x}, \widehat{\widetilde{x}})},
\end{aligned}
$$

where

$$
\begin{aligned}
& W_{1}(x, \widetilde{x}, \widehat{\widetilde{x}})=Q_{23}(\widetilde{x}, \widehat{\widetilde{x}}, \bullet, 0 ; q, r) Q_{31}(x, \bullet, \widetilde{x}, 0 ; r, p)-Q_{23}(\widetilde{x}, \widehat{\widetilde{x}}, 0,0 ; q, r) Q_{31}(x, \bullet, \widetilde{x}, \bullet ; r, p), \\
& W_{2}(x, \widetilde{x}, \widehat{\widetilde{x}})=Q_{23}(\widetilde{x}, \widehat{\widetilde{x}}, \bullet, 0 ; q, r) Q_{31}(x, 0, \widetilde{x}, 0 ; r, p)-Q_{23}(\widetilde{x}, \widehat{\widetilde{x}}, 0,0 ; q, r) Q_{31}(x, 0, \widetilde{x}, \bullet ; r, p), \\
& W_{3}(x, \widetilde{x}, \widehat{\widetilde{x}})=Q_{23}(\widetilde{x}, \widehat{\widetilde{x}}, \bullet \bullet \bullet ; q, r) Q_{31}(x, \bullet, \widetilde{x}, 0 ; r, p)-Q_{23}(\widetilde{x}, \widehat{\widetilde{x}}, 0, \bullet ; q, r) Q_{31}(x, \bullet, \widetilde{x}, \bullet ; r, p), \\
& W_{4}(x, \widetilde{x}, \widehat{\widetilde{x}})=Q_{23}(\widetilde{x}, \widehat{\widetilde{x}}, \bullet \bullet \bullet ; q, r) Q_{31}(x, 0, \widetilde{x}, 0 ; r, p)-Q_{23}(\widetilde{x}, \widehat{\widetilde{x}}, 0, \bullet ; q, r) Q_{31}(x, 0, \widetilde{x}, \bullet ; r, p), \\
& Z_{1}(x, \widehat{x}, \widehat{\widetilde{x}})=Q_{31}(\widehat{x}, \bullet, \widehat{\widetilde{x}}, 0 ; r, p) Q_{23}(x, \widehat{x}, \bullet, 0 ; q, r)-Q_{31}(\widehat{x}, 0, \widehat{\widetilde{x}}, 0 ; r, p) Q_{23}(x, \widehat{x}, \bullet \bullet \bullet ; q, r), \\
& Z_{2}(x, \widehat{x}, \widehat{\widetilde{x}})=Q_{31}(\widehat{x}, \bullet, \widehat{\widetilde{x}}, 0 ; r, p) Q_{23}(x, \widehat{x}, 0,0 ; q, r)-Q_{31}(\widehat{x}, 0, \widehat{\widetilde{x}}, 0 ; r, p) Q_{23}(x, \widehat{x}, 0, \bullet ; q, r), \\
& Z_{3}(x, \widehat{x}, \widehat{\widetilde{x}})=Q_{31}(\widehat{x}, \bullet, \widehat{\widetilde{x}}, \bullet ; r, p) Q_{23}(x, \widehat{x}, \bullet, 0 ; q, r)-Q_{31}(\widehat{x}, 0, \widehat{\widetilde{x}}, \bullet ; r, p) Q_{23}(x, \widehat{x}, \bullet, \bullet ; q, r), \\
& Z_{4}(x, \widehat{x}, \widehat{\widetilde{x}})=Q_{31}(\widehat{x}, \bullet, \widehat{\widetilde{x}}, \bullet ; r, p) Q_{23}(x, \widehat{x}, 0,0 ; q, r)-Q_{31}(\widehat{x}, 0, \widehat{\widetilde{x}}, \bullet ; r, p) Q_{23}(x, \widehat{x}, 0, \bullet ; q, r) \text {. }
\end{aligned}
$$

We use the symbol $\stackrel{\circ}{=}$ for equalities that should hold modulo an acceptable equation. For a set of equations the equality should be modulo the same equation, which we call $Q_{12}$.

Since $Q_{12}$ does not contain $y$, the Bäcklund condition $\widehat{\widetilde{y}}_{23} \stackrel{\circ}{=} \widehat{\widetilde{y}}_{31}$ implies three equations

$$
\begin{array}{lr}
y^{2}: & W_{1} Z_{3}-W_{3} Z_{1} \stackrel{\circ}{=} 0, \\
y^{1}: & W_{1} Z_{4}+W_{2} Z_{3}-W_{4} Z_{1}-W_{3} Z_{2} \stackrel{\circ}{=} 0 \\
y^{0}: & W_{2} Z_{4}-W_{4} Z_{2} \stackrel{\circ}{=} 0 .
\end{array}
$$

For a genuine eqBT these equations should not all be satisfied automatically but rather for at least one equation the LHS should factor with an acceptable equation as a factor.

\subsection{Deriving the Lax condition}

It is interesting to compare eqBT with conditions derived from a Lax pair. The starting point is still (24), but now we replace $y \rightarrow f / g$. Thus for example

$$
\begin{aligned}
\frac{\widehat{f}}{\widehat{g}} & =-\frac{Q_{23}(x, \widehat{x}, f / g, 0 ; q, r)}{Q_{23}(x, \widehat{x}, f / g, \bullet ; q, r)} \\
& =-\frac{Q_{23}(x, \widehat{x}, \bullet, 0 ; q, r) f+Q_{23}(x, \widehat{x}, 0,0 ; q, r) g}{Q_{23}(x, \widehat{x}, \bullet \bullet \bullet ; q, r) f+Q_{23}(x, \widehat{x}, 0, \bullet ; q, r) g}
\end{aligned}
$$


that is

$$
\left(\begin{array}{l}
\widehat{f} \\
\widehat{g}
\end{array}\right)=\mathcal{M}\left(\begin{array}{l}
f \\
g
\end{array}\right), \quad \mathcal{M}=\mu(x, \widehat{x})\left(\begin{array}{cc}
-Q_{23}(x, \widehat{x}, \bullet, 0 ; q, r) & -Q_{23}(x, \widehat{x}, 0,0 ; q, r) \\
Q_{23}(x, \widehat{x}, \bullet, \bullet ; q, r) & Q_{23}(x, \widehat{x}, 0, \bullet ; q, r)
\end{array}\right),
$$

where $\mu$ is a separation factor. Similarly

$$
\left(\begin{array}{c}
\tilde{f} \\
\widetilde{g}
\end{array}\right)=\mathcal{L}\left(\begin{array}{l}
f \\
g
\end{array}\right), \quad \mathcal{L}=\lambda(x, \widetilde{x})\left(\begin{array}{cc}
-Q_{31}(x, \bullet, \widetilde{x}, 0 ; r, p) & -Q_{31}(x, 0, \widetilde{x}, 0 ; r, p) \\
Q_{31}(x, \bullet, \widetilde{x}, \bullet ; r, p) & Q_{31}(x, 0, \widetilde{x}, \bullet ; r, p)
\end{array}\right) .
$$

Then the commutativity condition

$$
\left(\begin{array}{c}
\widetilde{f} \\
\widetilde{g}
\end{array}\right) \stackrel{\circ}{=}\left(\begin{array}{l}
\widehat{f} \\
\widehat{g}
\end{array}\right)^{\sim}
$$

implies

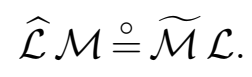

The entries of this matrix equation are

$$
\begin{aligned}
& (1,1): \quad \lambda(x, \widetilde{x}) \mu(\widetilde{x}, \widehat{\widetilde{x}}) W_{1} \stackrel{\circ}{=} \lambda(\widehat{x}, \widehat{\widetilde{x}}) \mu(x, \widehat{x}) Z_{1}, \\
& (1,2): \quad \lambda(x, \widetilde{x}) \mu(\widetilde{x}, \widehat{\widetilde{x}}) W_{2} \stackrel{\circ}{=} \lambda(\widehat{x}, \widehat{\widetilde{x}}) \mu(x, \widehat{x}) Z_{2} \text {, } \\
& (2,1): \quad \lambda(x, \widetilde{x}) \mu(\widetilde{x}, \widehat{\widetilde{x}}) W_{3} \stackrel{\circ}{=} \lambda(\widehat{x}, \widehat{\widetilde{x}}) \mu(x, \widehat{x}) Z_{3} \text {, } \\
& (2,2): \quad \lambda(x, \widetilde{x}) \mu(\widetilde{x}, \widehat{\widetilde{x}}) W_{4} \stackrel{\circ}{=} \lambda(\widehat{x}, \widehat{\widetilde{x}}) \mu(x, \widehat{x}) Z_{4} \text {, }
\end{aligned}
$$

using the previous notation. By taking ratios we get

$$
W_{j} / W_{k} \stackrel{\circ}{=} Z_{j} / Z_{k}, \quad \forall j, k,
$$

that is, polynomial equations

$$
W_{j} Z_{k}-W_{k} Z_{j} \stackrel{\circ}{=} 0, \quad \forall j, k .
$$

These equations should either vanish, or factorize with the desired equation as a factor.

When comparing (30) with (27) it is easy to verify that (30) implies (27). However, in the other direction one finds that in addition to

$$
W_{1} / W_{3} \stackrel{\circ}{=} Z_{1} / Z_{3}, \quad W_{2} / W_{4} \stackrel{\circ}{=} Z_{2} / Z_{4},
$$

from (27a), (27c), one finds

$$
\left(W_{4} / W_{3}-Z_{4} / Z_{3}\right)\left(W_{1} W_{4}-W_{3} W_{4}\right) \stackrel{\circ}{=} 0
$$

from (27b) after eliminating $Z_{1}, Z_{2}$. This suggests a possible new and different type of solution with $W_{1} W_{4}-W_{3} W_{4}=0$. This, however, does not work: for the equations under consideration the expression $W_{1} W_{4}-W_{3} W_{4}$ never vanishes, and furthermore it cannot be used to generate a quad equation, as it depends only on 3 of the 4 corner variables. Thus we conclude that eqBT and Lax generate the same equations or fail together. 


\subsection{BT for the results obtained}

Above we have described how the eqBT computations lead to equations (27) which should not hold automatically, but only after using $Q_{12}=0$, in other words, $\widehat{\widetilde{y}}_{23}-\widehat{\widetilde{y}}_{31}$ should factorize, with $Q_{12}(x, \widetilde{x}, \widehat{x}, \widehat{\widetilde{x}} ; p, q)$ as one of the factors. We can of course do these computations for all three choices of side equations:

1. back-left-front-right equations producing bottom and top equations,

2. bottom-back-top-front equations producing left and right equations,

3. bottom-left-top-right equations producing back and front equation.

We have done these computations for all equations found to have CAC (one representative per orbit) as listed in Appendix A. The results are given in Tables 1-3. In the table a "0" means the corresponding eqBT produces nothing, i.e., the equations (27) are satisfied automatically, a "1" means the desired equation is uniquely produced. The remaining cases have a "2" indicating that the polynomial produced by the BT has two factors that can be taken as genuine equations (i.e. they are acceptable by Definition 1 and do not depend on the auxiliary variable $y$ ). A plain "2" means the two possibilities differ only by a sign, " $2 c^{n}$ " means that the extra equation has degree $n$ dependence on the $c_{j}$, while $2^{*}$ stands for the cases where the two equations are essentially different and the extra equation is a version of $\widetilde{x} \widehat{x}-x \widehat{\widetilde{x}}$. Finally, if one of the resulting equations is not quadratic its degree is given as subscript.

Here are some examples

- For equation $\{58,10,10\}$ the bottom-back-top-front side equations produce for the left equation a rational expression whose numerator factorizes as

$$
y(\widetilde{x} \bar{x}-x \overline{\widetilde{x}})\left[c_{6}(q, r)\left(c_{2}(p, q) \overline{\widetilde{x}}+c_{5}(p, q) \bar{x}\right) \widetilde{x}+c_{5}(p, q)\left(c_{4}(p, q) \bar{x}+c_{6}(q, r) \overline{\widetilde{x}}\right) x\right]
$$

The factor in round brackets is the expected left equation. Note that for the alternate equation in square brackets the $q$ dependence is superfluous since the back equation should only depend on $r, p$.

- For equation $\{10,15,58\}$ the the bottom-back-top-front side equations produce

$$
y(\widetilde{x} \bar{x}-x \overline{\widetilde{x}})[\bar{x} \widetilde{x}+x \overline{\widetilde{x}}+r(x \widetilde{x}+\bar{x} \overline{\widetilde{x}})]
$$

The term in square brackets is the proper left equation, the extra factor $\widetilde{x} \bar{x}-x \overline{\widetilde{x}}$ is simpler and appears often. For this case the same type of result is found for right, back and front equations. All " $2 *$ " cases are like this.

- For $\{63,59,31\}-1$ eqBT produces the bottom equation and as an alternative, $x \widehat{x} \widehat{\widetilde{x}}+$ $x \widehat{x} \widetilde{x}+x \widetilde{x} \widehat{\widetilde{x}}+\widehat{x} \widetilde{x} \widehat{\widetilde{x}}$, while for the top equation the alternative is $y+\widetilde{y}+\widehat{y}+\widehat{\widetilde{y}}$. 


\begin{tabular}{|c|c|c|c|c|c|c|c|}
\hline Eqn & $\stackrel{0}{0}$ & 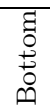 & 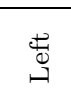 & $\begin{array}{l}\overrightarrow{0} \\
\overrightarrow{0.00} \\
\vec{c}\end{array}$ & 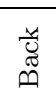 & $\begin{array}{l}\text { 节 } \\
\text { 至 }\end{array}$ & TET \\
\hline $63,10,10$ & 0 & 0 & 1 & 1 & 1 & 1 & no \\
\hline $62,10,10$ & 0 & 0 & 1 & 1 & 1 & 1 & no \\
\hline $58,10,10$ & 0 & 0 & $2 c^{2}$ & $2 c^{2}$ & 0 & 0 & no \\
\hline $42,10,10$ & 0 & 0 & 1 & 1 & 0 & 0 & no \\
\hline $10,10,10$ & 0 & 0 & 0 & 0 & 0 & 0 & no \\
\hline $43,10,10$ & 0 & 0 & 1 & 1 & 1 & 1 & no \\
\hline $61,10,10$ & 0 & 0 & 1 & 1 & $\overline{1}$ & 1 & no \\
\hline $60,10,10$ & 0 & 0 & 1 & 1 & 1 & 1 & no \\
\hline $56,10,10$ & 0 & 0 & $2 c^{1}$ & $2 c^{1}$ & 0 & 0 & no \\
\hline $53,10,10$ & 0 & 0 & 1 & 1 & 1 & 1 & yes \\
\hline $52,10,10$ & 0 & 0 & 1 & 1 & 1 & 1 & yes \\
\hline $05,10,10$ & 0 & 0 & 0 & 0 & 2 & 2 & yes \\
\hline
\end{tabular}

(a) eqBT for equations of type $\{\mathrm{X}, 10,10\}$.

\begin{tabular}{|c|c|c|c|c|c|c|c|}
\hline Eqn & $\stackrel{0}{\oplus}$ & 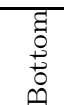 & 岕 & 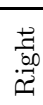 & $\begin{array}{l}\frac{\tilde{u}}{\tilde{D}} \\
\ddot{n}\end{array}$ & 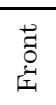 & TET \\
\hline $10,58,15$ & $2 c^{4}$ & $2 c^{4}$ & 0 & 0 & 0 & 0 & no \\
\hline $10,58,07$ & $2 c^{3}$ & $2 c^{3}$ & 0 & 0 & 0 & 0 & no \\
\hline $10,58,11$ & $2 c^{3}$ & $2 c^{3}$ & 0 & 0 & 0 & 0 & no \\
\hline $10,26,11$ & 1 & 1 & 0 & 0 & 0 & 0 & no \\
\hline $10,50,11$ & $2 c^{2}$ & $2 c^{2}$ & 0 & 0 & 0 & 0 & no \\
\hline $10,50,07$ & 1 & 1 & 0 & 0 & 0 & 0 & no \\
\hline $10,58,05$ & $2 c^{2}$ & $2 c^{2}$ & 0 & 0 & 0 & 0 & no \\
\hline $10,56,05$ & 1 & 1 & 0 & 0 & 0 & 0 & no \\
\hline $10,26,05$ & $2 c^{1}$ & $2 c^{1}$ & 0 & 0 & 0 & 0 & no \\
\hline $10,48,05$ & 0 & 0 & 0 & 0 & 0 & 0 & no \\
\hline
\end{tabular}

(b) eqBT for sub-cases of $\{10,58,15\}$.

Table 1: Results of eqBT for two classes of equations that can have free functions. "2" in a column means BT produces two possibilities, " $2 c^{n}$ " that the non-standard alternative has polynomial coefficients of degree $n$ in $c_{i}$. Only one representative of each orbit is listed. In the last column we indicate whether the triplet has the tetrahedron property.

\begin{tabular}{|c|c|c|c|c|c|c|c|}
\hline Eqn & ڤै & $\begin{array}{l}0 \\
\text { t. } \\
0\end{array}$ & 岕 & $\begin{array}{ll}\frac{\mathrm{t}}{\mathrm{vol}} \\
\overrightarrow{\mathrm{g}}\end{array}$ & 总 & 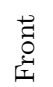 & TET \\
\hline $53,15,58(15)$ & 0 & 0 & 1 & 1 & 1 & 1 & yes \\
\hline $53,05,48(45)$ & 0 & 0 & 1 & 1 & 1 & 1 & yes \\
\hline $21,05,48(50)$ & 0 & 0 & 1 & 1 & 1 & 1 & yes \\
\hline $53,11,26(48)$ & 0 & 0 & 1 & 1 & 1 & 1 & yes \\
\hline $21,11,26(54)$ & 0 & 0 & 1 & 1 & 1 & 1 & yes \\
\hline $05,11,26(52)$ & 0 & 0 & 1 & 1 & $2^{*}$ & $2^{*}$ & yes \\
\hline $63,48,05(16)$ & 0 & 0 & 1 & 1 & 1 & 1 & no \\
\hline $58,48,05(42)$ & 0 & 0 & $2 c^{1}$ & $2 c^{1}$ & 0 & 0 & no \\
\hline $53,48,05(44)$ & 0 & 0 & 1 & 1 & 1 & 1 & yes \\
\hline $48,48,05(49)$ & 0 & 0 & 2 & 2 & 0 & 0 & yes \\
\hline $10,15,58(39)$ & 0 & 0 & $2^{*}$ & $2^{*}$ & $2^{*}$ & $2^{*}$ & yes \\
\hline
\end{tabular}

Table 2: eqBT for triplets with arbitrary functions listed in Figure 2. 


\begin{tabular}{|c|c|c|c|c|c|c|c|}
\hline Eqn & वे & 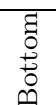 & $\underset{\mathrm{d}}{\stackrel{\mathrm{d}}{\mathrm{d}}}$ & $\begin{array}{l}\vec{n} \\
\overrightarrow{00} \\
\overrightarrow{\tilde{c}}\end{array}$ & 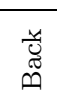 & $\begin{array}{l}\vec{Z} \\
\text { 总 }\end{array}$ & 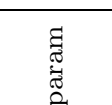 \\
\hline $63,63,63-1(58)$ & 1 & 1 & 1 & $\overline{1}$ & 1 & 1 & $p, q, r$ \\
\hline $63,59,31-1(55)$ & $2_{3}$ & $2_{1}$ & 1 & 1 & 1 & 1 & $p, q$ \\
\hline $63,63,63-2(17)$ & 1 & 1 & 1 & 1 & 1 & 1 & $p, q, r$ \\
\hline $63,59,31-2(56)$ & 1 & 1 & 1 & 1 & 1 & 1 & $p, q$ \\
\hline $63,58,15 \quad(40)$ & $2^{*}$ & $2^{*}$ & 1 & 1 & 1 & 1 & $p, q$ \\
\hline $27,31,59 \quad(53)$ & 1 & 1 & 1 & 1 & 1 & 1 & $r$ \\
\hline $26,15,59 \quad(43)$ & 1 & 1 & $2^{*}$ & $2^{*}$ & 1 & 1 & $r$ \\
\hline $10,15,58$ & 0 & 0 & $2^{*}$ & $2^{*}$ & $2^{*}$ & $2^{*}$ & $r$ \\
\hline $53,53,53 \quad(46)$ & 1 & 1 & 1 & 1 & 1 & 1 & $p, q, r$ \\
\hline $53,49,21 \quad(47)$ & 1 & 1 & 1 & 1 & 1 & 1 & $p, q$ \\
\hline
\end{tabular}

Table 3: eqBT for triplets in Figure 3, with specific $p, q, r$ dependency listed. As mentioned they are all reductions of $\{63,63,63-2\}$. For $\{63,59,31\}-1$ the subscript gives the degree of the other equation. All of these equations have the tetrahedron property. Note that the reduction $\{10,15,58\}$ can have free $c_{i}$ functions and therefore it fails some eqBTs, which is indicated by the 0 entries.

\section{Summary}

All the equations discussed here satisfy the consistency-around-the-cube condition. The equations on the consistency cube can also provide a Lax pair or produce a Bäcklund transformation in which the "side equations" may generate the bottom and top equations. It turns out that many of the equations produce a fake Lax pair or equivalently an empty eqBT. From the tables we can also read that some equations have the tetrahedron property and still fail with respect to eqBT. Furthermore some equations have ambiguous eqBT, generating two possible equations. If such a triplet does not completely fail in any direction we may have an example of partial integrability.

\section{Discussion}

We have searched for all homogeneous quadratic triplets of multilinear irreducible equations that satisfy the Consistency-Around-a-Cube condition with uniform embedding (translation invariance of quad equations). The results were given in Appendix A and classified in Section 4. The three equations forming the triplet were allowed to have different forms, while in the usual setup the bottom equation is given and the other equations are obtained from that by definite variable and parameter changes. In that sense our approach is close to that of Boll $[3,5]$.

The ansatz for the equations contained coefficients that depended on the two lattice parameters associated with the quadrilateral in question. The results can be divided into two classes: those that cannot have arbitrary dependence on the two lattice parameters 
and those that can. The arbitrary functions can sometimes be eliminated by a gauge transformation (that preserves uniformity) and therefore the possibility of free functions is essential. The results can be arranged into sub-cases, some relations are given in Figures 2-3. The equations that cannot have free functions are all reductions of $Q 3(\delta=0)$ of the ABS list [2].

Recently it has been noted that some equations that pass the CAC test can have fake Lax pairs [6], and independently the possibility of "weak Lax pairs" has also been observed [8]. In order to characterize the equations further we studied how the Bäcklund transformation works on these equations. The result are given in Tables 1-3. One finds that all equations that can have free functions also have some failing BTs (or equivalently some fake Lax pairs). This is natural since it is not possible to generate a free function from equations that do not contain them. Note that some of the equations with failing eqBTs do nevertheless have the tetrahedron property.

From our results we infer the following:

1. The CAC condition can only be a necessary condition of integrability. It cannot be sufficient as many equations with failing eqBTs pass this test.

2. The CAC condition may be sufficient if accompanied with some other type of condition, but the tetrahedron condition is not enough for that purpose.

3. We conjecture that if there is a unique BT for each direction of the cube then that should be sufficient for integrability. Non-uniqueness in some directions may be a signal of partial integrability while failure of the BT in one or more directions suggests non-integrability.

4. Integrability may be lost during reductions. But note that the reduction here does not change dimension but just means simplifying the equation by some limit of the coefficient(s).

\section{A Results of the search}

We will now list the CAC equation triplets within the category of equations studied in this paper, namely those that are quadratic and acceptable by Definition 1 . We list them following the order of the search process which was based on the triplet code. In Section 4 they are grouped according to the "highest equation" and its reductions. During the search process sub-case inclusion was not considered except for obvious reductions. If the triplet found is a member of a symmetry orbit of more than one entry we also list the orbit. 


\section{A.1 $\{10,10,10\}$}

The triplet

$$
\begin{aligned}
& c_{2}(p, q) \widetilde{x} \widehat{x}+c_{4}(p, q) x \widehat{\widetilde{x}}=0, \\
& c_{2}(q, r) \widehat{x} \bar{x}+c_{4}(q, r) x \overline{\widehat{x}}=0, \\
& c_{2}(r, p) \bar{x} \widetilde{x}+c_{4}(r, p) x \overline{\widetilde{x}}=0,
\end{aligned}
$$

satisfies the CAC condition without any constraints on the parameters $c_{2}, c_{4}$. However, by the gauge transform (11) we can take the triplet into the simple form

$$
\begin{aligned}
& \widetilde{x} \widehat{x}-x \widehat{\widetilde{x}}=0, \\
& \widehat{x} \bar{x}-x \overline{\widehat{x}}=0, \\
& \bar{x} \widetilde{x}-x \overline{\widetilde{x}}=0,
\end{aligned}
$$

with

$$
\overline{\widehat{x}}=\frac{\widetilde{x} \widehat{x} \bar{x}}{x^{2}},
$$

which does not have the tetrahedron property.

$$
\text { A.2 }\{X, 10,10\}, X \neq 10
$$

This category contains the triplet $\{\mathbf{6 3}, \mathbf{1 0}, \mathbf{1 0}\}$ and its special cases.

$$
\begin{aligned}
x \widetilde{x} c_{1}(p, q)+\widetilde{x} \widehat{x} c_{2}(p, q)+\widehat{x} \widehat{\widetilde{x}} c_{3}(p, q)+x \widehat{\widetilde{x}} c_{4}(p, q)+x \widehat{x} c_{5}(p, q)+\widetilde{x} \widehat{\widetilde{x}} c_{6}(p, q) & =0, \\
\widehat{x} \bar{x}-x \widehat{\widehat{x}} & =0 \\
\bar{x} \widetilde{x}-x \overline{\widetilde{x}} & =0
\end{aligned}
$$

That is, a completely arbitrary homogeneous quadratic equation is MDC with the side equations as given above. The triply shifted $x$ is given by

$$
\overline{\widehat{x}}=-\frac{c_{5}(p, q) x \bar{x} \widehat{x}+c_{1}(p, q) x \widetilde{x} \bar{x}+c_{2}(p, q) \widetilde{x} \widehat{x} \bar{x}}{c_{4}(p, q) x^{2}+c_{6}(p, q) x \widetilde{x}+c_{3}(p, q) x \widehat{x}},
$$

and thus (33) has TET in the sub-case $c_{2}(p, q)=c_{4}(p, q)=0$, with code $\{53,10,10\}$.

The sub-cases of $\{63,10,10\}$ arrange themselves into orbits as follows: 


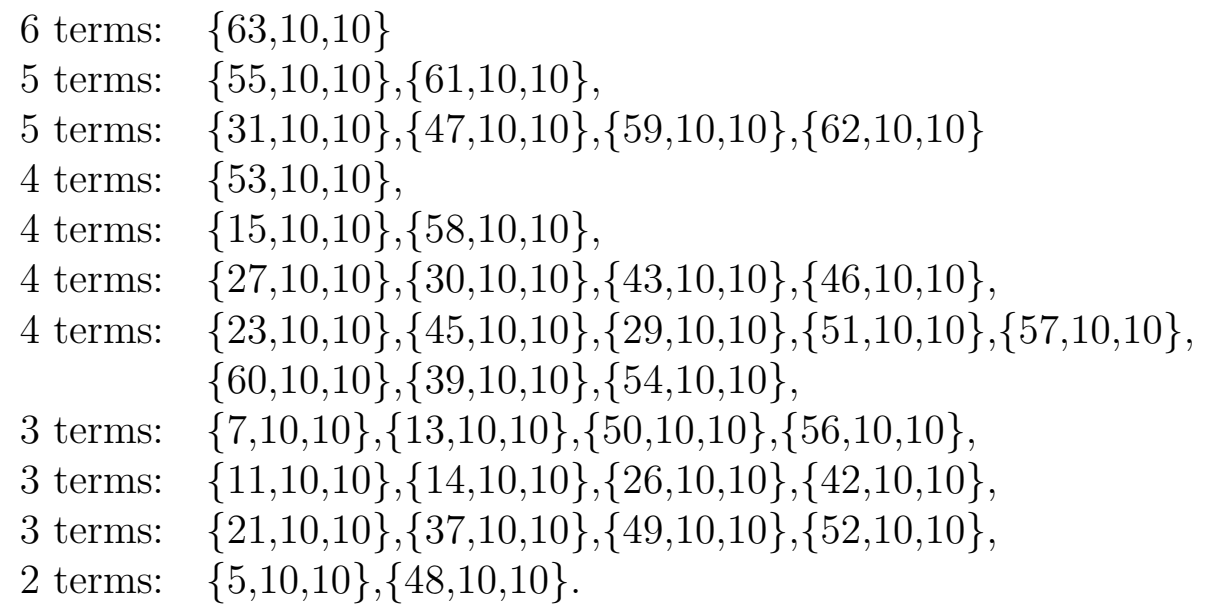

In solving the CAC equations for these triplet codes we were usually led to conditions on the $c_{2}, c_{4}$ terms of the back and left equation. For $\{63,10,10\}$ it is not possible to have any free functions in the side equations, but as the $\{58,10,10\}$ (13) case shows, with smaller number of terms in the bottom equation we have more freedom in the side equations but that freedom can be eliminated by gauge.

\section{A.3 $\{10, X, Y\}, X, Y \neq 10$}

In this case we arrange the bottom equation to be the simple one. The main result in this category is $\{\mathbf{1 0}, \mathbf{5 8}, \mathbf{1 5}\}$ :

$$
\begin{aligned}
\widehat{x} \widetilde{x}-x \widehat{\widetilde{x}} & =0 \\
x \bar{x} c_{5}(q, r)+x \overline{\widehat{x}} c_{4}(q, r)+\bar{x} \widehat{x} c_{2}(q, r)+\overline{\widehat{x}} \widehat{x} c_{6}(q, r) & =0 \\
x \bar{x} c_{1}(r, p)+x \bar{x} c_{4}(r, p)+\bar{x} \widetilde{x} c_{2}(r, p)+\overline{\widetilde{x}} \widetilde{x} c_{3}(r, p) & =0 .
\end{aligned}
$$

The triply shifted quantity is given by

$$
\overline{\widehat{x}}=\bar{x} \frac{\left(c_{1}(r, p) x+c_{2}(r, p) \widetilde{x}\right)\left(c_{5}(q, r) x+c_{2}(q, r) \widehat{x}\right)}{\left(c_{4}(r, p) x+c_{3}(r, p) \widetilde{x}\right)\left(c_{4}(q, r) x+c_{6}(q, r) \widehat{x}\right)} .
$$

This has sub-cases when one or two $c_{i}$ in either or both equations vanish while keeping the equations irreducible. Missing $c_{j}$ terms sometimes allow a more general bottom equation, but that freedom can be gauged away. The sub-case orbits are as follows: 


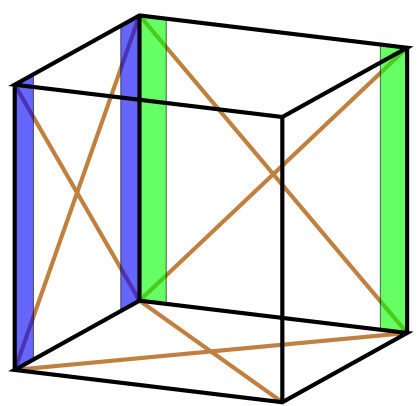

(34)

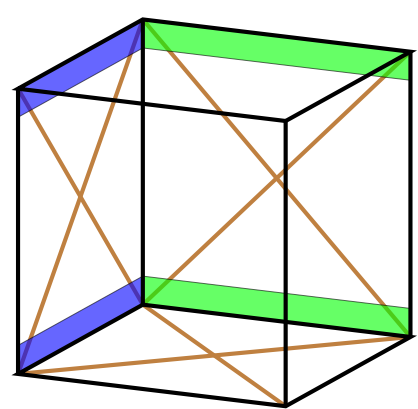

(39)

Figure 4: A diagrammatic way of describing the nonzero terms in the triplets (34) and (39): each line or band connecting two points corresponds to their product term in the equation.

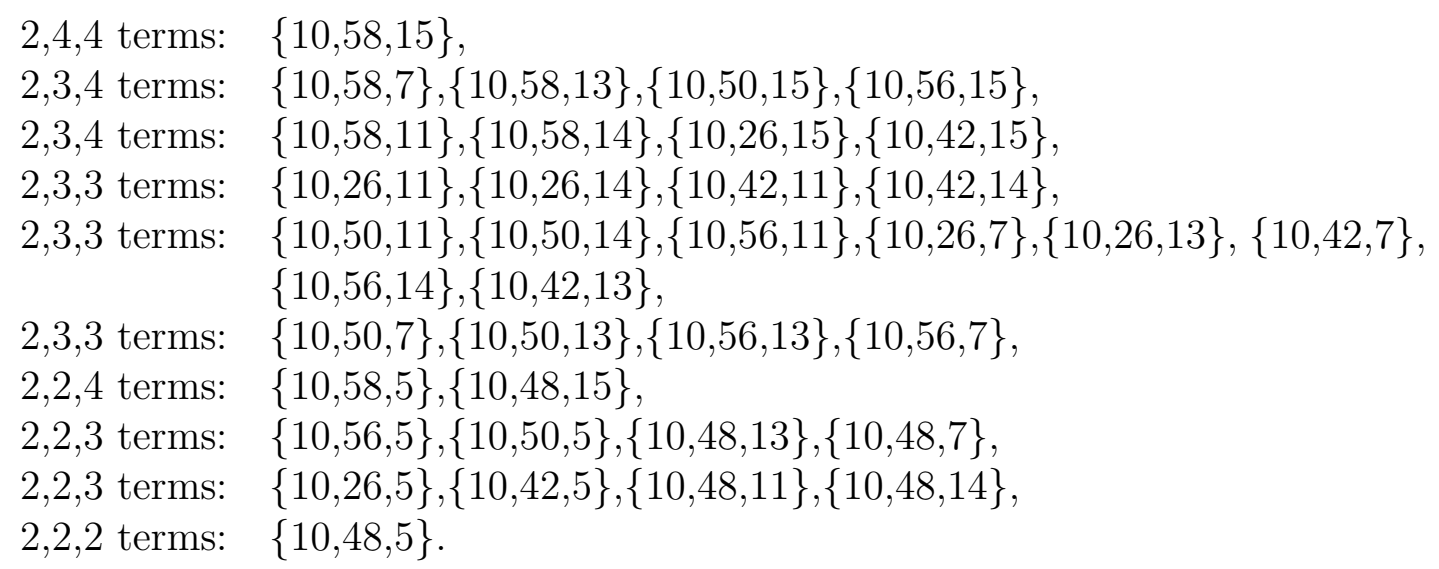

The number of terms in each equation is given in the first column, but that list is not ordered because some reflections exchange back and left equations. The sub-cases belonging to $\{\mathrm{X}, 10,10\}$ are excluded.

$\{\mathbf{1 0 , 1 5 , 5 8}\}$ In this category there is also an equation that is not a sub-case of $(34)$ :

$$
\begin{array}{r}
\widehat{x} \widetilde{x}+x \widehat{\widetilde{x}} c_{4}(p, q)=0, \\
\bar{x} \widehat{x}+x \overline{\widehat{x}}+x \widehat{x} g(r)^{-1}+\bar{x} \bar{x} f(r)^{-1}=0 \\
\bar{x} \widetilde{x}+x \overline{\widetilde{x}}+x \widetilde{x} f(r)+\bar{x} \overline{\widetilde{x}} g(r)=0,
\end{array}
$$

with

$$
\overline{\widehat{\widetilde{x}}}=-\frac{\widetilde{x} \widehat{x} f(r)}{\bar{x} c_{4}(p, q) g(r)}
$$

This cannot be a sub-case of (14) because the equations do not contain the same shifted $x$ variables. We can use gauge transformation to simplify this further. Choosing the gauge so that (37) simplifies to

$$
\overline{\widehat{\widetilde{x}}}=-\frac{\widetilde{x} \widehat{x}}{\bar{x}}
$$


the equations get the form (after redefining $r$ )

$$
\begin{array}{r}
\widehat{x} \widetilde{x}-x \widehat{\widetilde{x}}=0, \\
r(\bar{x} \widehat{x}+x \overline{\widehat{x}})+x \widehat{x}+\bar{x} \bar{x}=0 \\
\bar{x} \widetilde{x}+x \overline{\widetilde{x}}+r(x \widetilde{x}+\bar{x} \bar{x})=0
\end{array}
$$

For an intuitive understanding of the difference between (34) and (39) we can look at the Figure 4. Note also that in (35) $\bar{x}$ is in the numerator while in (38) it is in the denominator.

\section{A.4 $\{58,15, X\}, X \neq 10$}

Only $\{\mathbf{6 3}, \mathbf{5 8}, \mathbf{1 5}\}$ survives, all other cases lead to equations that factorize.

$$
\begin{aligned}
p\left(1-q^{2}\right)(x \widehat{x}+\widetilde{x} \widehat{\widetilde{x}})-q\left(1-p^{2}\right)(x \widetilde{x}+\widehat{x} \widehat{\widetilde{x}})+\left(p^{2}-q^{2}\right)(x \widehat{\widetilde{x}}+\widehat{x} \widetilde{x}) & =0, \\
q(x \bar{x}+\widehat{x} \bar{x})+x \bar{x}+\bar{x} \widehat{x} & =0, \\
p(x \bar{x}+\widetilde{x} \bar{x})+x \overline{\widetilde{x}}+\bar{x} \widetilde{x} & =0 . \\
\overline{\widehat{x}}=\frac{\bar{x}(-p \widehat{x}+q \widetilde{x})}{p \widetilde{x}-q \widehat{x}} . &
\end{aligned}
$$

\section{A.5 $\{15,58, X\}, X \neq 10,15,58$}

According to (8a) we have by tilde-hat reflection and rotation $\{15,58, X\} \rightarrow\{58, \theta X, 15\} \rightarrow$ $\{15,58, \theta X\}$. Thus $\{15,58, X\}$ and $\{58,15, Y\}$, are not related by this reflection and may have different types of solutions.

The main solution in this category is $\{\mathbf{5 3}, \mathbf{1 5}, \mathbf{5 8}\}$, and after a gauge transformation the equation satisfying $\mathrm{CAC}$ can be written as

$$
\begin{aligned}
c_{6}(p, q) \widehat{\widetilde{x}} \widetilde{x}+c_{3}(p, q) \widehat{x} \widehat{\widetilde{x}}+c_{1}(p, q) x \widetilde{x}+c_{5}(p, q) x \widehat{x} & =0, \\
r(x \widehat{x}+\bar{x} \bar{x})+x \bar{x}+\bar{x} \widehat{x} & =0 \\
r(x \widetilde{x}+\bar{x} \bar{x})+x \overline{\widetilde{x}}+\bar{x} \widetilde{x} & =0
\end{aligned}
$$

The triply shifted variable is

$$
\overline{\widehat{\widetilde{x}}}=-\bar{x} \frac{c_{1}(p, q) \widetilde{x}+c_{5}(p, q) \widehat{x}}{c_{6}(p, q) \widetilde{x}+c_{3}(p, q) \widehat{x}}
$$

thus it has TET. All other equations in this category that satisfy CAC are sub-cases of the above, or lead to equations that factorize. The orbits for the sub-cases are

4 terms: $\{53,15,58\}$,

3 terms: $\{21,15,58\},\{37,15,58\},\{49,15,58\},\{52,15,58\}$,

2 terms: $\quad\{5,15,58\},\{48,15,58\}$. 


\section{A.6 $\{15, X, Y\}, X, Y \notin\{10,58\}$ or $\{58, X, Y\}, X, Y \notin\{10,15\}$}

By the tilde-hat reflection we have $\{15, X, Y\} \rightarrow\{58, \theta Y, \theta X\}$ and therefore there is oneto-one correspondence between the sets $\{15, X, Y\}$ and $\left\{58, X^{\prime}, Y^{\prime}\right\}$ and we only need to discuss one of them.

There are types two solutions, the first one is $\{\mathbf{5 8 , 4 8 , 5}\}$ given by

$$
\begin{aligned}
c_{4}(p, q)\left(\sigma_{1} \widetilde{x} \widehat{x}+x \widehat{\widetilde{x}}\right)+c_{5}(p, q)\left(\sigma_{2} x \widehat{x}+\widetilde{x} \widehat{\widetilde{x}}\right) & =0, \\
c_{5}(q, r) x \bar{x}+c_{6}(q, r) \widehat{x} \bar{x} & =0 \\
x \bar{x}-\sigma_{1} \sigma_{2} \widetilde{x} \overline{\widetilde{x}} & =0
\end{aligned}
$$

with

$$
\overline{\widehat{x}}=\frac{x \bar{x} c_{5}(q, r)}{\widehat{x} c_{6}(q, r)} \frac{c_{4}(p, q) x+c_{5}(p, q) \widetilde{x}}{c_{4}(p, q) \sigma_{2} \widetilde{x}+\sigma_{1} c_{6}(p, q) x} .
$$

One can change some of the signs by gauge but one cannot eliminate the freedom in $c_{i}$. This does not have TET except if $c_{4}(p, q)=0$, in which case it becomes $\{48,48,5\}(49)$. The reflected case is $\{15,48,5\}$.

$\{\mathbf{2 6 , 1 5 , 5 9}\}$ The second solution is, after gauge and rotation

$$
\begin{aligned}
x \widehat{x}+x \widehat{\widetilde{x}}+\widetilde{x} \widehat{x} & =0, \\
r(x \widehat{x}+\overline{\widehat{x}} \bar{x})+x \widehat{x}+\bar{x} \widehat{x} & =0, \\
\left(r^{2}-1\right) x \bar{x}+r^{2}(x \overline{\widetilde{x}}+\bar{x} \widetilde{x})+r(x \widetilde{x}+\overline{\widetilde{x}} \bar{x}) & =0, \\
\overline{\widehat{\widetilde{x}}}=\frac{\widehat{x}(\bar{x}-r \widetilde{x})}{r \bar{x}} &
\end{aligned}
$$

The triplet (43) is symmetric under bar reversal and tilde reversal, but not under hatreversal and therefore there are also the corresponding solution within three reversed categories. The orbit is $\{15,59,26\},\{15,62,42\},\{58,11,31\},\{58,14,47\}$.

\section{A.7 $\{53, X, Y\}, X, Y \notin\{10,15,58\}$}

There are five solutions in this category and they all have TET.

\section{$\{53,48,5\}$}

$$
\begin{aligned}
c_{5}(p, q)\left(x \widehat{x}-\sigma_{1} \sigma_{2} \widehat{\widetilde{x}} \widetilde{x}\right)+c_{1}(p, q)\left(x \widetilde{x}-\sigma_{1} \sigma_{3} \widehat{x} \widehat{\widetilde{x}}\right) & =0, \\
x \bar{x}-\sigma_{3} \overline{\widehat{x}} \widehat{x} & =0, \\
x \bar{x}-\sigma_{2} \overline{\widetilde{x}} \widetilde{x} & =0,
\end{aligned}
$$

where $\sigma_{j}^{2}=1$. The signs $\sigma_{j}$ can be changed by gauge.

$$
\overline{\widehat{x}}=\frac{\bar{x}\left(c_{1}(p, q) \sigma_{2} \widehat{x}+c_{5}(p, q) \sigma_{3} \widetilde{x}\right)}{\sigma_{1}\left(c_{1}(p, q) \widetilde{x}+c_{5}(p, q) \widehat{x}\right)} .
$$




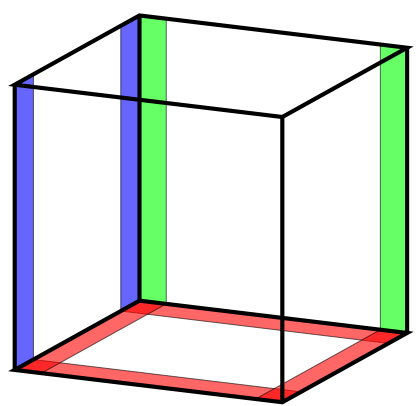

(44)

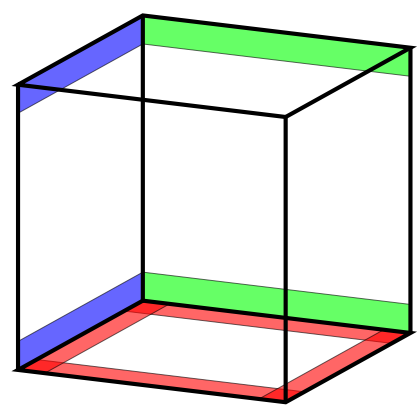

$(45)$

Figure 5: Diagrams for the nonzero terms in the triplets (44) and (45).

$\{53,5,48\}$

$$
\begin{aligned}
\left.c_{1}(p, q) x \widetilde{x}+c_{3}(p, q) \widehat{x} \widehat{\widetilde{x}}+c_{5}(p, q) x \widehat{x}+c_{6}(p, q) \widetilde{x} \widehat{\widetilde{x}}\right) & =0, \\
x \widehat{x}+\overline{\widehat{x}} \bar{x} & =0, \\
x \widetilde{x}+\widetilde{\widetilde{x}} \bar{x} & =0, \\
\overline{\widehat{\widetilde{x}}}=-\bar{x} \frac{c_{1}(p, q) \widetilde{x}+c_{5}(p, q) \widehat{x}}{c_{6}(p, q) \widetilde{x}+c_{3}(p, q) \widehat{x}} &
\end{aligned}
$$

The sub-case orbits are as for $\{53,15,58\}(41)$.

The difference between (44) and (45) is illustrated in Figure 5.

\section{$\{53,53,53\}$}

$$
\begin{aligned}
p\left(x \widehat{x} \delta_{p}+\widetilde{x} \widehat{\widetilde{x}} \rho_{p}\right)-q\left(x \widetilde{x} \delta_{q}+\widehat{x} \widehat{\widetilde{x}} \rho_{q}\right) & =0, \\
q\left(x \bar{x} \delta_{q}+\widehat{x} \bar{x} \rho_{q}\right)-r\left(x \widehat{x} \delta_{r}+\bar{x} \widehat{x} \rho_{r}\right) & =0, \\
r\left(x \widetilde{x} \delta_{r}+\bar{x} \overline{\widetilde{x}} \rho_{r}\right)-p\left(x \bar{x} \delta_{p}+\widetilde{x} \overline{\widetilde{x}} \rho_{p}\right) & =0,
\end{aligned}
$$

with triply shifted variable

$$
\overline{\widehat{\widetilde{x}}}=\frac{\bar{x} \widehat{x} \delta_{p} p\left(\rho_{q} \delta_{q} q^{2}-\rho_{r} \delta_{r} r^{2}\right)+\bar{x} \widetilde{x} \delta_{q} q\left(\rho_{r} \delta_{r} r^{2}-\rho_{p} \delta_{p} p^{2}\right)+\widehat{x} \widetilde{x} \delta_{r} r\left(\rho_{p} \delta_{p} p^{2}-\rho_{q} \delta_{q} q^{2}\right)}{\widetilde{x} \rho_{p} p\left(\rho_{r} \delta_{r} r^{2}-\rho_{q} \delta_{q} q^{2}\right)+\widehat{x} \rho_{q} q\left(\rho_{p} \delta_{p} p^{2}-\rho_{r} \delta_{r} r^{2}\right)+\bar{x} \rho_{r} r\left(\rho_{q} \delta_{q} q^{2}-\rho_{p} \delta_{p} p^{2}\right)} .
$$

We have added here some coefficients $\delta_{\alpha}$ and $\rho_{\alpha}$ and if they are nonzero they can be gauged to 1 , which yields $H 3(\delta=0)$ of the ABS list. If a $\rho_{\alpha}$ vanishes we get a rotation of $\{53,49,21\}$, if a $\delta_{\alpha}$ vanishes we get a rotation of its reflection $\{53,52,37\}$, and if $\delta_{\alpha}=\rho_{\alpha}=0$ for some $\alpha$ we get a rotation of a special case of $\{53,48,05\}$.

\section{$\{53,49,21\}$}

$$
\begin{aligned}
q(x \widetilde{x}+\widehat{x} \widehat{\widetilde{x}})-p(x \widehat{x}+\widetilde{x} \widehat{\widetilde{x}}) & =0, \\
x \widehat{x}-q(x \bar{x}+\widehat{x} \bar{x}) & =0, \\
x \widetilde{x}-p(x \bar{x}+\widetilde{x} \bar{x}) & =0
\end{aligned}
$$




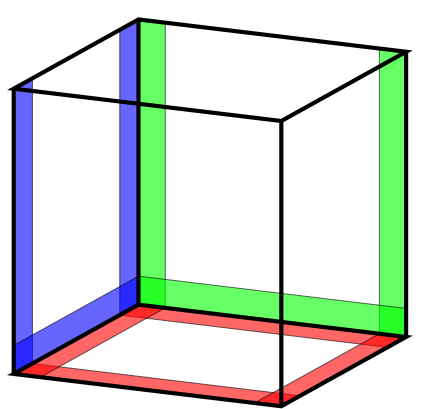

(47)

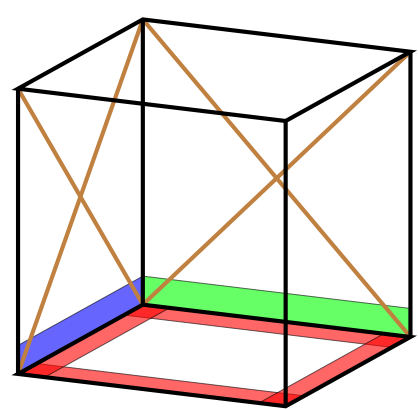

(48)

Figure 6: Diagrams for the nonzero terms in the triplets (47) and (48).

$$
\overline{\widehat{x}}=\frac{p q \bar{x}(q \widehat{x}-p \widetilde{x})+\left(p^{2}-q^{2}\right) \widehat{x} \widetilde{x}}{p q(p \widehat{x}-q \widetilde{x})} .
$$

There is also the reflected case $\{53,52,37\}$.

\section{$\{53,11,26\}$}

$$
\begin{aligned}
\left.c_{1}(p, q) x \widetilde{x}+c_{3}(p, q) \widehat{x} \widehat{\widetilde{x}}+c_{5}(p, q) x \widehat{x}+c_{6}(p, q) \widetilde{x} \widehat{\widetilde{x}}\right) & =0, \\
x \widehat{x}+\bar{x} \widehat{x}+\widehat{\widehat{x}} x & =0, \\
x \widetilde{x}+\bar{x} \widetilde{x}+\overline{\widetilde{x}} x & =0, \\
\overline{\widehat{x}}=-\bar{x} \frac{c_{1}(p, q) \widetilde{x}+c_{5}(p, q) \widehat{x}}{c_{6}(p, q) \widetilde{x}+c_{3}(p, q) \widehat{x}} . &
\end{aligned}
$$

Note that $\overline{\widehat{\widetilde{x}}}$ is the same as for $\{53,5,48\}$ (45). There is also the reflected case $\{53,14,42\}$. Sub-case orbits are as for $\{53,15,58\}(15)$.

The difference between (47) and (48) is illustrated in Figure 6.

\section{A.8 $\{5, X, Y\}$ or $\{48, X, Y\} X, Y \notin\{10,15,53,58\}$}

By the tilde-hat reflection we have $\{5, X, Y\} \rightarrow\{48, \theta Y, \theta X\}$. The solutions in this category are as follows:

\section{$\{48,48,05\}$}

$$
\begin{aligned}
x \widehat{x}-\sigma_{1} \widetilde{x} \widehat{x} & =0, \\
c_{5}(p, q) x \bar{x}+c_{6}(p, q) \widehat{x} \bar{x} & =0, \\
x \bar{x}-\sigma_{2} \widetilde{x} \bar{x}= & 0, \\
\overline{\widehat{x}}=-\sigma_{1} \sigma_{2} \widetilde{x} \frac{\bar{x} c_{5}(q, r)}{\widehat{x} c_{6}(p, q)} . &
\end{aligned}
$$

The signs can be controlled by gauge. In this orbit there is also $\{05,05,48\}$. 
$\{21,05,48\}$

$$
\begin{aligned}
c_{1}(p, q) x \widetilde{x}+c_{3}(p, q) \widehat{x} \widehat{\widetilde{x}}+c_{5}(p, q) x \widehat{x} & =0, \\
x \widehat{x}+\bar{x} \bar{x} & =0, \\
x \widetilde{x}+\bar{x} \overline{\widetilde{x}} & =0, \\
\overline{\widehat{x}}=-\bar{x} \frac{\widetilde{x} c_{1}(q, r)+\widehat{x} c_{5}(p, q)}{\widehat{x} c_{3}(p, q)} &
\end{aligned}
$$

The full orbit of reflected cases is $\{21,5,48\},\{37,5,48\},\{49,5,48\},\{52,5,48\}$.

\section{$\{63,48,5\}$}

$$
\begin{aligned}
& c_{1}(p, q)\left(x \widetilde{x}-\sigma_{1} \sigma_{3} \widehat{x} \widehat{\widetilde{x}}\right)+c_{5}(p, q)\left(x \widehat{x}-\sigma_{1} \sigma_{2} \widehat{\widetilde{x}} \widetilde{x}\right)+c_{4}(p, q)\left(\widehat{x} \widetilde{x}-\sigma_{1} x \widehat{\widetilde{x}}\right)=0, \\
& x \bar{x}-\sigma_{3} \overline{\widehat{x}} \hat{x}=0, \\
& x \bar{x}-\sigma_{2} \overline{\widetilde{x}} \widetilde{x}=0, \\
& \overline{\widehat{\widetilde{x}}}=\frac{x \bar{x}\left(c_{1}(p, q) \sigma_{3} \widehat{x}+c_{4}(p, q) x+c_{5}(p, q) \sigma_{2} \widetilde{x}\right)}{\sigma_{1} \sigma_{2} \sigma_{3}\left(c_{1}(p, q) x \widetilde{x}+c_{4}(p, q) \widehat{x} \widetilde{x}+c_{5}(p, q) x \widehat{x}\right)} .
\end{aligned}
$$

The sign $\sigma_{1}$ cannot be eliminated by gauge.

$\{5,11,26\}$

$$
\begin{gathered}
c_{1}(p, q) x \widetilde{x}+c_{3}(p, q) \widehat{x} \widehat{\widetilde{x}}=0, \\
x \widehat{x}+\bar{x} \widehat{x}+x \overline{\widehat{x}}=0, \\
x \widetilde{x}+\widetilde{x} \bar{x}+x=0, \\
\overline{\widetilde{x}}=0, \widetilde{x} \bar{x} c_{1}(q, r) \\
\widehat{\widehat{x}}=-\frac{}{\widehat{x} c_{3}(p, q)} .
\end{gathered}
$$

The orbit is $\{5,11,26\},\{48,11,26\},\{5,14,42\},\{48,14,42\}$.

\section{A.9 The rest: $\{X, Y, Z\}, X, Y, Z \notin\{5,10,15,53,58\}$}

\section{$\{27,31,59\}$}

$$
\begin{aligned}
x \widehat{x}+x \widetilde{x}+x \widehat{\widetilde{x}}+\widehat{x} \widetilde{x} & =0, \\
\left(1-r^{2}\right) x \bar{x}+(x \bar{x}+\bar{x} \widehat{x})+r(x \widehat{x}+\bar{x} \bar{x}) & =0, \\
\left(r^{2}-1\right) x \bar{x}+r^{2}(x \overline{\widetilde{x}}+\bar{x} \widetilde{x})+r(x \widetilde{x}+\bar{x} \bar{x}) & =0, \\
\overline{\widehat{x}}=\frac{r^{2} \bar{x} \widetilde{x}-r \widehat{x} \widetilde{x}+\bar{x} \widehat{x}}{r \bar{x}} . &
\end{aligned}
$$

There are altogether 4 different reflections of this triplet: $\{27,31,59\},\{43,31,62\},\{30,47,59\}$, $\{46,47,62\}$. 
$\{21,11,26\}$

$$
\begin{aligned}
x \widehat{x} c_{5}(p, q)+x \widetilde{x} c_{1}(p, q)+\widehat{x} \widehat{\widetilde{x}} c_{3}(p, q) & =0, \\
x \widehat{x}+x \overline{\widehat{x}}+\bar{x} \widehat{x} & =0, \\
x \widetilde{x}+x \overline{\widetilde{x}}+\bar{x} \widetilde{x} & =0 . \\
\overline{\widehat{x}}=-\frac{\widetilde{x} \bar{x} c_{1}(q, r)+\widehat{x} \bar{x} c_{5}(p, q)}{\widehat{x} c_{3}(p, q)} &
\end{aligned}
$$

There are 8 elements in the orbit $\{11,26,21\},\{11,26,37\},\{11,26,49\},\{11,26,52\},\{14,42,21\}$, $\{14,42,37\},\{14,42,49\},\{14,42,52\}$;

The remaining cases were the difficult ones to solve. In the category $\{63,59,31\}$ there are two solutions:

\section{$\{63,59,31\}-1$}

$$
\begin{gathered}
(q-p)(x \widehat{\widetilde{x}}+\widehat{x} \widetilde{x})-p(x \widetilde{x}+\widehat{x} \widehat{\widetilde{x}})+q(x \widehat{x}+\widehat{\widetilde{x}} \widetilde{x})=0 \\
q x \widehat{x}+(x \bar{x}+\overline{\widehat{x}} \widehat{x})+(x \overline{\widehat{x}}+\bar{x} \widehat{x})=0, \\
p x \widetilde{x}+(x \bar{x}+\overline{\widetilde{x}} \widetilde{x})+(x \overline{\widetilde{x}}+\widetilde{x} \bar{x})=0 \\
\overline{\widetilde{\widetilde{x}}}=\bar{x}+\frac{(p-q) \widehat{x} \widetilde{x}}{\widehat{x}-\widetilde{x}} .
\end{gathered}
$$

Note that the back and left equations are not related by a cyclic variable change.

\section{$\{63,59,31\}-2$}

$$
\begin{aligned}
& \left(p^{2}-q^{2}\right)(x \widehat{\widetilde{x}}+\widehat{x} \widetilde{x})+\left(p^{2}-1\right) q(x \widetilde{x}+\widehat{x} \widehat{\widetilde{x}})-p\left(q^{2}-1\right)(x \widehat{x}+\widehat{\widetilde{x}} \widetilde{x})=0, \\
& \left(q^{2}-1\right) x \widehat{x}+q(x \bar{x}+\bar{x} \widehat{x})+x \overline{\widehat{x}}+\bar{x} \widehat{x}=0, \\
& \left(p^{2}-1\right) x \widetilde{x}+p(x \bar{x}+\overline{\widetilde{x}} \widetilde{x})+x \overline{\widetilde{x}}+\bar{x} \widetilde{x}=0, \\
& \overline{\widehat{\widetilde{x}}}=\frac{\left(q^{2}-p^{2}\right) \widehat{x} \widetilde{x}+\bar{x}(q \widetilde{x}-p \widehat{x})}{p \widetilde{x}-q \widehat{x}} .
\end{aligned}
$$

There are also corresponding two reflected cases $\{63,62,47\}$.

$\{\mathbf{6 3 , 6 3 , 6 3}\}$ In the category $\{63,63,63\}$ there are also two solutions. The preliminary way of writing the result, after a gauge transformation, is the form

$$
\begin{aligned}
& c(q)(x \widetilde{x}+\widehat{x} \widehat{\widetilde{x}})-c(p)(x \widehat{x}+\widehat{\widetilde{x}} \widetilde{x})-p(x \widehat{\widetilde{x}}+\widehat{x} \widetilde{x})+q(x \widehat{\widetilde{x}}+\widehat{x} \widetilde{x})=0, \\
& c(r)(x \widehat{x}+\bar{x} \bar{x})-c(q)(x \bar{x}+\widehat{\widehat{x}} \hat{x})-q(x \widehat{\widehat{x}}+\bar{x} \widehat{x})+r(x \widehat{\widehat{x}}+\bar{x} \widehat{x})=0, \\
& c(r)(x \widetilde{x}+\bar{x} \overline{\tilde{x}})-c(p)(x \bar{x}+\overline{\widetilde{x}} \widetilde{x})-p(x \overline{\widetilde{x}}+\bar{x} \widetilde{x})+r(x \overline{\widetilde{x}}+\bar{x} \widetilde{x})=0 .
\end{aligned}
$$


where we still have the conditions

$$
c(\alpha)^{2}=\alpha^{2}+a_{1} \alpha+a_{0}, \quad \alpha \in\{p, q, r\} .
$$

If $a_{1}$ and/or $a_{0}$ are nonzero the result is awkward, but we can simplify the result by a transformation $p=f(P), q=f(Q), r=f(R)$. However, in order to connect with known results we we want the coefficient of the $(x \widehat{\widetilde{x}}+\widehat{x} \widetilde{x})$ be $\left(P^{2}-Q^{2}\right)$ after the transformation and a possible overall multiplication. For simplicity let us redefine $a_{0}=a_{1}^{2} / 4-a_{2}^{2}$. There are two possibilities:

$\{63,63,63\}-1$ If $a_{2}=0$ then by a simple translation we get $c(\alpha)=\epsilon(\alpha) \alpha$, where $\epsilon(\alpha)^{2}=1$, but this sign can be eliminated by gauge. In order to connect with known results we redefine $p \mapsto 1 / p, q \mapsto 1 / q, r \mapsto 1 / r$ after which we get

$$
\begin{gathered}
(q-p)(x \widehat{\widetilde{x}}+\widehat{x} \widetilde{x})-q(x \widehat{x}+\widehat{\widetilde{x}} \widetilde{x})+p(x \widetilde{x}+\widehat{x} \widehat{\widetilde{x}})=0, \\
(r-q)(x \overline{\widehat{x}}+\bar{x} \widehat{x})-r(x \bar{x}+\overline{\widehat{x}} \widehat{x})+q(x \widehat{x}+\bar{x} \bar{x})=0, \\
(p-r)(x \overline{\widetilde{x}}+\widetilde{x} \bar{x})-p(x \widetilde{x}+\overline{\widetilde{x}} \bar{x})+r(x \bar{x}+\widetilde{x} \bar{x})=0 \\
\overline{\widehat{\widetilde{x}}}=\frac{r \bar{x}(\widehat{x}-\widetilde{x})+q \widehat{x}(\widetilde{x}-\bar{x})+p \widetilde{x}(\bar{x}-\widehat{x})}{r(\widehat{x}-\widetilde{x})+q(\widetilde{x}-\bar{x})+p(\bar{x}-\widehat{x})}
\end{gathered}
$$

This is in fact $\mathrm{Q} 1(\delta=0)$ in the ABS list.

$\{63,63,63\}-2 \quad$ A different solution is obtained if $a_{2} \neq 0$. Then by transforming

$$
p=\frac{\left(-a_{1}+2 a_{2}\right) P^{2}+a_{1}+2 a_{2}}{2 P^{2}-2},
$$

etc. one finds

$$
c(P)=\epsilon_{P} a_{2} \frac{2 P}{P^{2}-1}, \text { and }(p-q) \rightarrow-2 a_{2} \frac{P^{2}-Q^{2}}{\left(P^{2}-1\right)\left(Q^{2}-1\right)},
$$

and after clearing denominators (and renaming $P \rightarrow p)$ we get $Q 3(\delta=0)$ of the ABS list:

$$
\begin{aligned}
& \left(q^{2}-p^{2}\right)(x \widehat{\widetilde{x}}+\widehat{x} \widetilde{x})-p\left(q^{2}-1\right)(x \widehat{x}+\widehat{\widetilde{x}} \widetilde{x})+\left(p^{2}-1\right) q(x \widetilde{x}+\widehat{x} \widehat{\widetilde{x}})=0, \\
& \left(r^{2}-q^{2}\right)(x \widehat{\widehat{x}}+\bar{x} \widehat{x})-q\left(r^{2}-1\right)(x \bar{x}+\widehat{\widehat{x}} \widehat{x})+\left(q^{2}-1\right) r(x \widehat{x}+\bar{x} \widehat{x})=0, \\
& \left(p^{2}-r^{2}\right)(x \overline{\widetilde{x}}+\widetilde{x} \bar{x})-r\left(p^{2}-1\right)(x \widetilde{x}+\widetilde{\widetilde{x}} \bar{x})+\left(r^{2}-1\right) p(x \bar{x}+\widetilde{x} \overline{\tilde{x}})=0,
\end{aligned}
$$

with

$$
\overline{\widehat{\widetilde{x}}}=\frac{\bar{x} \widehat{x} p\left(q^{2}-r^{2}\right)+\bar{x} \widetilde{x} q\left(r^{2}-p^{2}\right)+\widehat{x} \widetilde{x} r\left(p^{2}-q^{2}\right)}{\bar{x} r\left(q^{2}-p^{2}\right)+\widehat{x} q\left(p^{2}-r^{2}\right)+\widetilde{x} p\left(r^{2}-q^{2}\right)} .
$$

Note that the difference between Q1 and Q3 arises from the factorization properties of the $c(\alpha)$ coefficient in (57). 


\section{Acknowledgment}

I would like to thank Da-jun Zhang for useful comments on the manuscript. All computations were done using the REDUCE computer algebra system [7]

\section{References}

[1] V. E. Adler. Bäcklund transformation for the Krichever-Novikov equation. Internat. Math. Res. Notices, (1):1-4, 1998.

[2] V. E. Adler, A. I. Bobenko, and Yu. B. Suris. Classification of integrable equations on quad-graphs. The consistency approach. Comm. Math. Phys., 233(3):513-543, 2003.

[3] R Boll. Classification of 3D consistent quad-equations. J. Nonlinear Math. Phys., 18(3):337-365, 2011.

[4] R Boll. Corrigendum: "Classification of 3D consistent quad-equations." J. Nonlinear Math. Phys., 19(4):1292001, 3.pp, 2012.

[5] R. Boll. Classification and Lagrangian Structure of 3D Consistent Quad-Equations. https://depositonce.tu-berlin.de/handle/11303/3599. Thesis 130pp. 2012.

[6] S Butler and M Hay. Simple identification of fake Lax pairs. arXiv:1311.2406, 2013.

[7] A. C. Hearn and R. Schöpf. REDUCE User's Manual, Free Version. https://reduce-algebra.sourceforge.io/manual/manual.html. 2019.

[8] J Hietarinta and C Viallet. Weak Lax pairs for lattice equations. Nonlinearity, 25(7):1955-1966, 2012.

[9] Levi D and Winternitz P. Continuous symmetries of difference equations. J. Phys. A: Math. Gen., 39(2):R1, 2006. arXiv:nlin/0502004

[10] Nijhoff F, Ramani A, Grammaticos B, and Ohta Y. On discrete Painlevé equations associated with the lattice KdV systems and the Painlevé VI equation. Stud. Appl. Math., 106(3):261-314, 2001.

[11] C.-M. Viallet. Algebraic entropy for lattice equations. arXiv:math-ph/0609043v2, 2006.

[12] C.-M. Viallet. Integrable lattice maps: $Q_{V}$, a rational version of $Q_{4}$. Glasg. Math. J., 51(A):157-163, 2009. 